\title{
Cancer susceptibility and embryonic lethality in Mob1a/1b double-mutant mice
}

\author{
Miki Nishio,, Koichi Hamada, ${ }^{1}$ Kohichi Kawahara, ${ }^{1}$ Masato Sasaki, ${ }^{2,3}$ Fumihito Noguchi, ${ }^{4}$ \\ Shuhei Chiba, ${ }^{5}$ Kensaku Mizuno, ${ }^{5}$ Satoshi O. Suzuki, ${ }^{6}$ Youyi Dong, ${ }^{7}$ Masaaki Tokuda, ${ }^{7}$ \\ Takumi Morikawa, ${ }^{1}$ Hiroki Hikasa, ${ }^{1}$ Jonathan Eggenschwiler, ${ }^{8}$ Norikazu Yabuta, ${ }^{9}$ Hiroshi Nojima, ${ }^{9}$ \\ Kentaro Nakagawa, ${ }^{10}$ Yutaka Hata, ${ }^{10}$ Hiroshi Nishina, ${ }^{11}$ Koshi Mimori, ${ }^{12}$ Masaki Mori, ${ }^{12,13}$ \\ Takehiko Sasaki, ${ }^{2,14}$ Tak W. Mak, ${ }^{3}$ Toru Nakano, ${ }^{15}$ Satoshi Itami, ${ }^{4}$ and Akira Suzuki ${ }^{1,2}$ \\ ${ }^{1}$ Division of Cancer Genetics, Medical Institute of Bioregulation, Graduate School of Medical Sciences, Kyushu University, Fukuoka, Japan. \\ ${ }^{2}$ Global Centers of Excellence Program, Akita University Graduate School of Medicine, Akita, Japan. ${ }^{3}$ The Campbell Family Institute for Cancer Research, \\ University Health Network, Toronto, Ontario, Canada. ${ }^{4}$ Department of Regenerative Dermatology, Graduate School of Medicine, Osaka University, Suita, \\ Osaka, Japan. ${ }^{5}$ Department of Biomolecular Sciences, Graduate School of Life Sciences, Tohoku University, Sendai, Miyagi, Japan. \\ ${ }^{6}$ Department of Neuropathology, Graduate School of Medical Sciences, Kyushu University, Fukuoka, Japan. ${ }^{7}$ Department of Cell Physiology, \\ Faculty of Medicine, Kagawa University, Miki-cho, Kagawa, Japan. ${ }^{8}$ Department of Molecular Biology, Princeton University, Princeton, New Jersey, USA. \\ ${ }^{9}$ Department of Molecular Genetics, Research Institute for Microbial Diseases, Osaka University, Suita, Osaka, Japan. ${ }^{10}$ Department of Medical Biochemistry \\ and ${ }^{11}$ Department of Developmental and Regenerative Biology, Graduate School of Medicine, Tokyo Medical and Dental University, Tokyo, Japan. \\ ${ }^{12}$ Department of Surgery, Kyushu University, Beppu Hospital, Beppu, Oita, Japan. ${ }^{13}$ Department of Gastroenterological Surgery, Graduate School of Medicine, \\ Osaka University, Suita, Osaka, Japan. ${ }^{14}$ Department of Medical Biology, Akita University Graduate School of Medicine, Akita, Japan. \\ ${ }^{15}$ Department of Pathology, Medical School and Graduate School of Frontier Biosciences, Osaka University, Suita, Osaka, Japan.
}

\begin{abstract}
Mps one binder 1a (MOB1A) and MOB1B are key components of the Hippo signaling pathway and are mutated or inactivated in many human cancers. Here we show that intact Mob1a or Mob1b is essential for murine embryogenesis and that loss of the remaining WT Mob1 allele in $M o b 1 a^{\Delta / \Delta} 1 b^{t r /+}$ or $M o b 1 a^{\Delta /+} 1 b^{t r / t r}$ mice results in tumor development. Because most of these cancers resembled trichilemmal carcinomas, we generated doublemutant mice bearing tamoxifen-inducible, keratinocyte-specific homozygous-null mutations of Mob1a and $M o b 1 b$ ( $k \mathrm{DKO}$ mice). $k \mathrm{DKO}$ mice showed hyperplastic keratinocyte progenitors and defective keratinocyte terminal differentiation and soon died of malnutrition. $k$ DKO keratinocytes exhibited hyperproliferation, apoptotic resistance, impaired contact inhibition, enhanced progenitor self renewal, and increased centrosomes. Examination of Hippo pathway signaling in $k D K O$ keratinocytes revealed that loss of $M o b 1 a / b$ altered the activities of the downstream Hippo mediators LATS and YAP1. Similarly, YAP1 was activated in some human trichilemmal carcinomas, and some of these also exhibited MOB1A/1B inactivation. Our results clearly demonstrate that MOB1A and MOB1B have overlapping functions in skin homeostasis, and exert their roles as tumor suppressors by regulating downstream elements of the Hippo pathway.
\end{abstract}

\section{Introduction}

Tissue homeostasis requires a balance of cell proliferation, apoptosis, and differentiation. During tissue development, progenitor cells divide for a limited number of times before exiting the cell cycle and undergoing terminal differentiation (1). When progenitor cells proliferate inappropriately and/or undergo abnormal differentiation, cancer can result (2).

Skin is a tissue that begins as a single-layered epithelium made up of keratinocytes that later stratify and differentiate into hair follicles (HFs) or interfollicular epidermis (IFE). Disruption of signals balancing growth and differentiation in keratinocytes can initiate skin cancers. The most common skin cancer is basal cell carcinoma (BCC), the nodular subtype of which originates from HFs. Trichilemmal carcinomas are also HF-derived skin cancers, but are much more rare and show characteristic abnormalities of outer hair root sheath differentiation (3). Although impairment of the Sonic Hedgehog (SHH) intracellular signaling pathway is now well established as the cause of BCC $(4,5)$,

Authorship note: Koichi Hamada and Kohichi Kawahara contributed equally to this work. Satoshi Itami and Akira Suzuki contributed equally as co-senior authors. Conflict of interest: The authors have declared that no conflict of interest exists. Citation for this article: J Clin Invest. doi:10.1172/JCI63735. the molecular mechanism underlying the development of trichilemmal carcinomas is unknown.

The evolutionarily conserved Hippo signaling pathway was first identified as regulating the cell proliferation and apoptosis-controlling organ size in Drosopbila $(6,7)$. In mammals, the canonical Hippo pathway includes the following: Neurofibromin 2 (NF2) (8), the Mammalian STE20-like protein (MST) kinases (9), large tumor suppressor homolog (LATS) and Nuclear Dbf2-related (NDR) kinases (10), the adaptor proteins SAV1 (WW45) (11) and MOB (12), and their downstream transcriptional coactivators YAP1 and its paralog transcriptional coactivator with PDZ-binding motif (TAZ, WWTR1) (13). All of these molecules (except YAP1 and TAZ) have been implicated as tumor suppressors. For example, mice deficient in Lats1 or Mst1/2 develop various cancers (14,15), SAV1 and MOB are mutated in human cancer cell lines $(16,17)$, and Hippo components are reduced in human cancer samples (18). Homozygous-null mutant mice lacking Nf2, Mst1/2, Sav1, Lats2, or Yap1 are all embryonic lethal $(15,19-22)$, precluding study of these mediators in tumorigenesis. To complicate matters, multiple homologs of each mammalian Hippo element exist, and a noncanonical Hippo pathway influences the localization and activity of canonical Hippo components $(15,23)$. Thus, the precise physiological functions of Hippo elements in mammals remain obscure. 
A Mob1a $a^{N+1 b^{t / n t}} \quad$ B intercrosses

Mob1a $^{+/+} 1 \mathrm{~b}^{\mathrm{t} / \mathrm{tr}}$

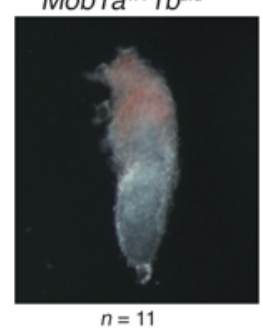

Mob1 $a^{N+1}+b^{t r t r}$

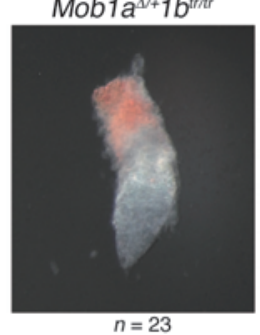

Absorbed

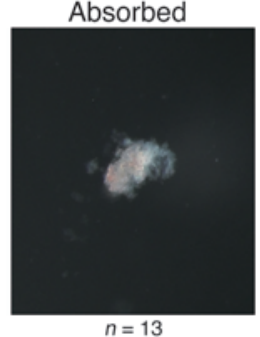

D
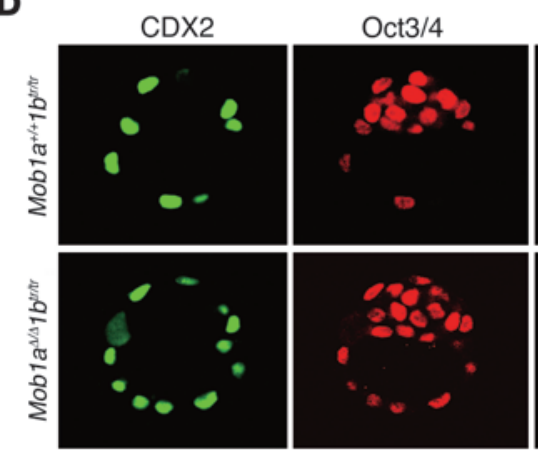

Sox2
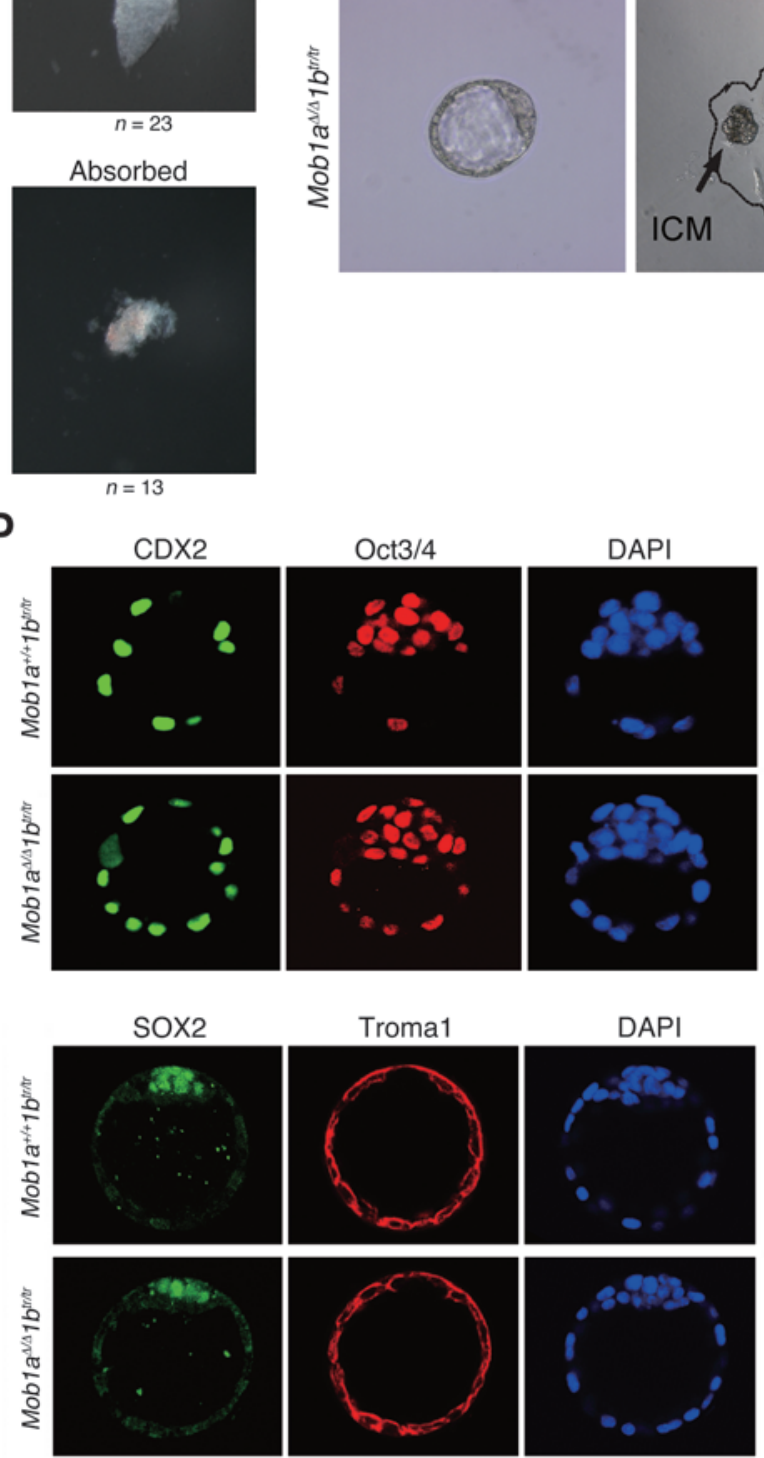

Day 8
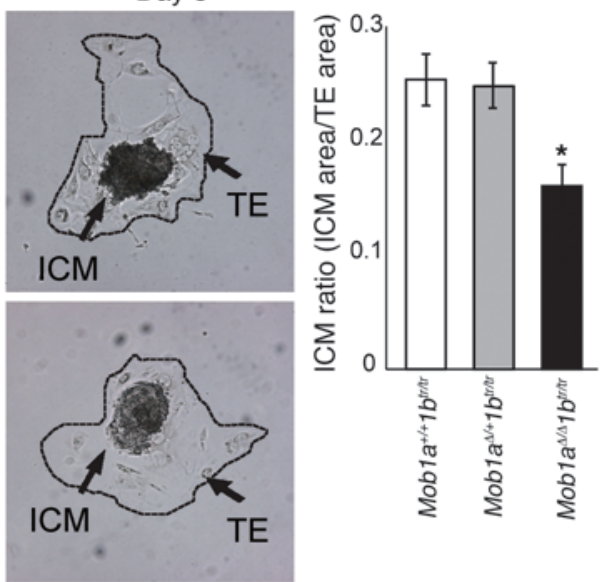

C

ERCreMob1 $a^{t / n} 1 b^{t r t r}$ EB

(\% of control)
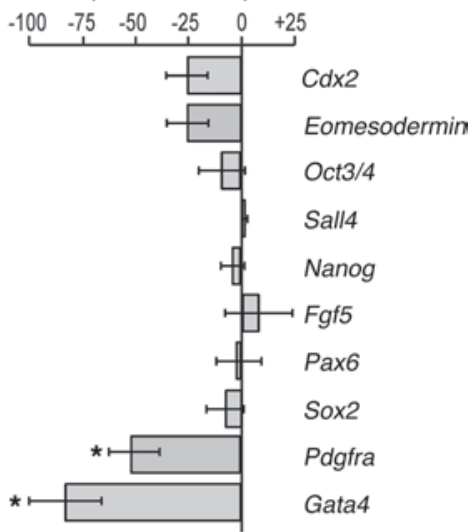
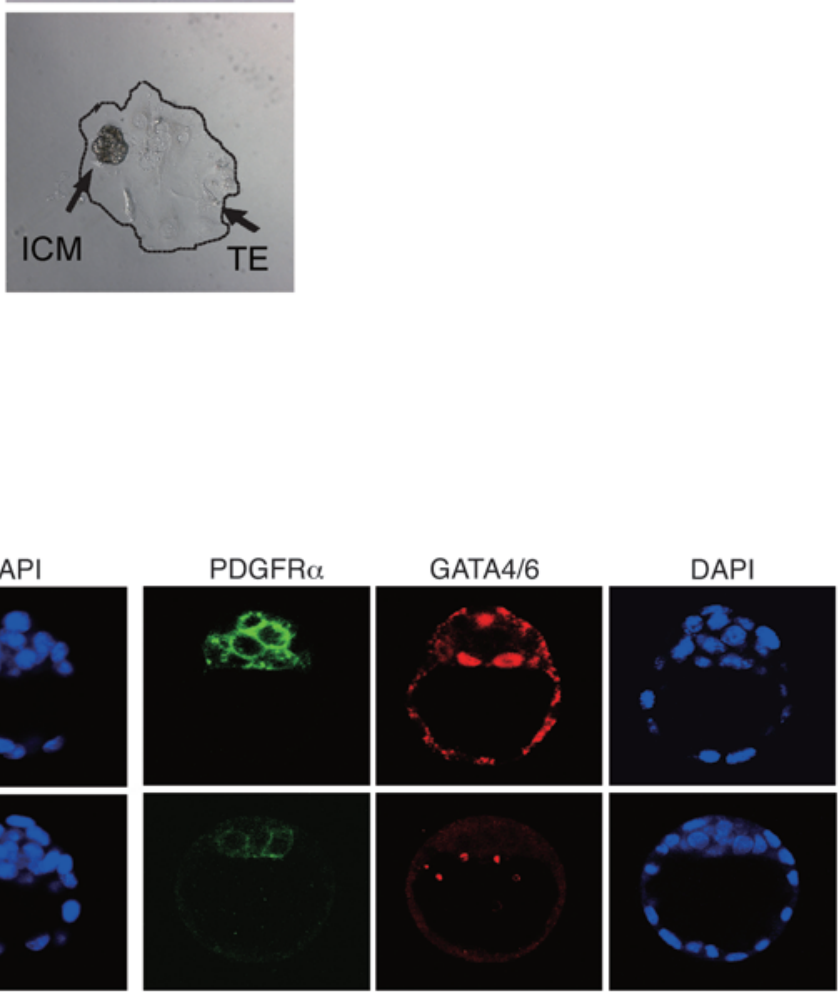

NANOG
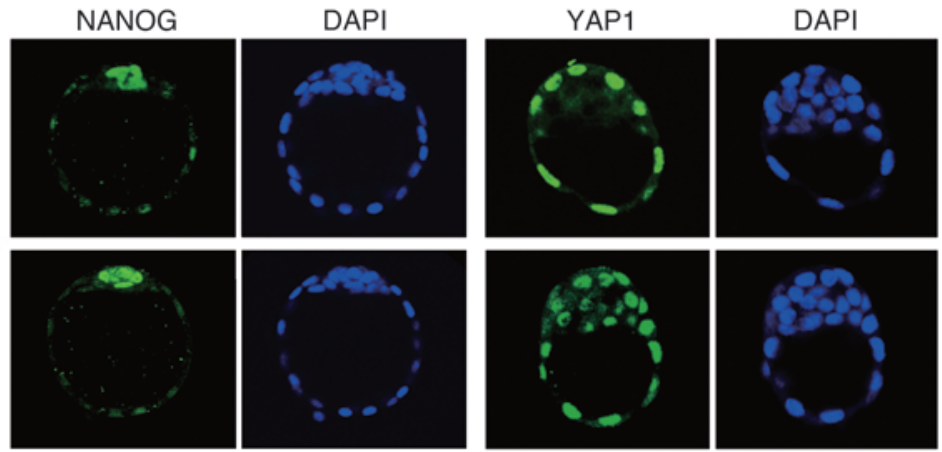


\section{Figure 1}

Loss of Mob1a/1b impairs mouse embryo survival. (A) Morphologies of representative E6.5 embryos of the indicated genotypes from Mob1 $a^{\Delta /+1} b^{t r / t r}$ intercrosses. Mob1 $a^{\Delta / \Delta} 1 b^{t r / t r}$ embryos were absorbed after implantation. (B) Left: photographs of E3.5 blastocysts of the indicated genotypes on day 0 and after culture for 8 days. The ICM is surrounded by TE giant cells. Right: mean ICM ratio (ICM area/TE area) \pm SEM plotted for blastocysts of the indicated genotypes $(n=8 /$ group); ${ }^{*} P<0.01$. (C) Quantitative PCR determination of mRNAs for the indicated genes in EBs generated from ERCreMob1 $a^{\text {fl/tl } 1} b^{\text {tr/tr }}$ ES cells. ES cells were cultured with/without tamoxifen for 2 days, and control and mutant EBs were generated using the hanging drop method after culture for 3 days (Cdx2, Eomesodermin, Oct3/4, Sall4) or 4 days (Nanog, Fgf5, Pax6, Sox2, Pdgfra, Gata4). mRNA levels in the mutant are expressed as the percentage increase over control values; ${ }^{*} P<0.05$. (D) Immunostaining to detect the indicated proteins in blastocysts of the indicated genotypes. Nuclei were visualized with DAPI. Results shown are representative of at least 3 independent trials and at least 3 mice/group. Data are presented as the mean \pm SEM, and $P$ values were determined using the 2-tailed Student's $t$ test.

$M O B 1$ was originally shown in yeast to regulate mitotic exit and modulate cytokinesis (24-29). In Drosophila, dMOB1 (MATS) overexpression is tumor suppressive, while loss of $d M O B 1$ triggers tumor development (17). In humans, $7 \mathrm{MOB}$ homologs (hMOB1A-B, 2A-C, 3, 4, where MOB indicates Mps one binder) have been identified (30), and hMOB1A (hMATS1, MOBKL1B) and hMOB1B (hMATS2, MOBKL1A) share 95\% amino acid identity. Although no apparent functional domain has been found in hMOB1A/1B, only these $2 \mathrm{MOB}$ proteins can bind to and activate LATS1/2 (31). In vitro, hMOB1A overexpression inhibits cell proliferation, while suppression of hMOB1B or hMOB1A mediated by shRNA or siRNA increases proliferation (30) or impairs mitotic exit (32), respectively. In vivo, the $b M O B 1 A$ gene is mutated in melanoma and breast cancer cell lines, and its expression is downregulated in human colorectal and non-small-cell lung cancers $(17,33$, 34). However, the normal function of hMOB1 proteins in vivo is unknown. To assess whether MOB1 is an important functional component of mammalian Hippo signaling and to determine whether MOB1 is truly a tumor suppressor in vivo, we generated and characterized double-mutant mice lacking Mob1a and Mob1b.

\section{Results}

Embryonic lethality of Mob1a/1b-null mutant mice. We first generated single-mutant mice bearing a null mutation of Mob1a $\left(M_{0 b} a^{\Delta / \Delta}\right)$ or a trapped mutation of Mob1b (Mob1 $\left.b^{t r / t r}\right)$ (Supplemental Figure 1, A-D; supplemental material available online with this article; doi:10.1172/JCI63735DS1) but no abnormalities were observed in morphology, body weight, histology, or life span (Supplemental Figure 1E and data not shown). We then attempted to generate double-homozygous-null mutant $\left(M o b 1 a^{\Delta / \Delta} 1 b^{t r / t r}\right)$ mice, but no viable pups were obtained (Supplemental Table 1), indicating that complete loss of Mob1 is embryonic lethal. We next analyzed embryos from $M o b 1 A^{\Delta /+} 1 b^{t r / t r}$ intercrosses at various time points during gestation. By E6.5, although decidua were formed, 28\% of embryos were absorbed too severely to genotype (Figure $1 \mathrm{~A}$ and Table 1). Thus, MOB1A and MOB1B have overlapping functions, and MOB1 is essential for postimplantation embryogenesis.

To pinpoint the embryonic defect, we obtained individual blastocysts (E3.5) from Mob1 $a^{\Delta /+} 1 b^{t r / t r}$ intercrosses and cultured them for 8 days. Mob1 $a^{\Delta / \Delta} 1 b^{t r / t r}$ blastocysts appeared normal at E3.5, but showed growth failure of the inner cell mass (ICM) by day 8 (Figure 1B). In contrast, the trophectoderm (TE) of Mob1 $1 a^{\Delta / \Delta} 1 b^{t r / t r}$ blastocysts developed normally.

To create conditional tamoxifen-inducible Mob1 mutants, we generated ERCreMob1 $a^{f l / f l} 1 b^{t r / t r}$ ES cells and established embryoid bodies (EBs) using the hanging drop method. Control and mutant EBs were cultured for 2 days with (or without) tamoxifen to delete Mob1 and then grown without leukemia inhibitory factor (LIF) for 3-4 days. Quantitative RT-PCR examination showed that Gata4 and Pdgfra mRNAs (essential for primitive endoderm formation) were markedly suppressed in Mob1 $a^{\Delta / \Delta} 1 b^{t r / t r}$ EBs, whereas $C d x 2$ and Eomesodermin mRNAs (essential for TE) were only slightly inhibited (Figure 1C). Levels of Oct3/4 (Pou5f1) and Sall4 mRNAs (essential for ICM), as well as Nanog, Fgf5, Pax6, and Sox2 mRNAs (essential for primitive ectoderm), were normal in the doublemutant EBs. Immunostaining of double-mutant blastocysts with antibodies recognizing CDX2 or Troma-1 (TE markers), NANOG or SOX2 (primitive ectoderm markers), or Oct3/4 confirmed that the levels of these proteins were also normal in the absence of Mob1. However, levels of GATA4 and PDGFRA proteins (primitive endoderm markers) were greatly reduced in the mutants (Figure 1D). Moreover, YAP1, which is usually expressed only in the nuclei of TE cells, was abnormally activated and expressed in the nuclei of ICM cells (Figure 1D). Thus, Mob1 $a^{\Delta / \Delta} 1 b^{t r / t r}$ embryos have a defect in primitive endoderm formation.

Mob1 $a^{\Delta /+} 1 b^{\text {tr/tr }}$ and Mob1 $a^{\Delta / \Delta} 1 b^{\text {tr/+}}$ mice show increased susceptibility to tumorigenesis. Mob1 $a^{\Delta /+} 1 b^{t r / t r}$ and Mob1 $a^{\Delta / \Delta} 1 b^{t r /+}$ mice were born alive and were healthy and fertile, but $52 \%$ of them had dental malocclusion (Supplemental Figure 2A). In addition, 25\% of these heterozygotes exhibited disequilibrium due to disorganized inner ear hair bundles in the cochlear organ of Corti (Supplemental Figure 2B and Supplemental Video 1), and 31\% showed histological evidence of increased trabeculae in femurs (Supplemental Figure 2C).

Because mutation or reduced expression of MOB1A occurs in cancer patients (33), we monitored tumorigenesis in Mob1 $a^{\Delta /+} 1 b^{t r / t r}$, Mob1 $a^{\Delta / \Delta} 1 b^{t r /+}$, and Mob1 $a^{\Delta /+} 1 b^{t r /+}$ (control) mice over 70 weeks. Various tumor types arose spontaneously in $100 \%$ of Mob1 $a^{\Delta /+} 1 b^{t r / t r}$ and Mob1 $a^{\Delta / \Delta} 1 b^{t r /+}$ mice, but in only $4 \%$ of controls (Figure 2, A and B, and Table 2). Southern blotting confirmed the loss of the WT Mob1 allele in all tumors (Figure 2C). In most mutants, skin tumors in the form of facial swellings (Figure 2A) were observed. Benign exos-

\section{Table 1}

Viability of embryos of the indicated Mob1a genotypes from Mob1 $a^{\Delta /+} 1 b^{\text {tr/tr }}$ intercrosses at the indicated stages of embryogenesis

\begin{tabular}{lccccc}
\hline \multicolumn{5}{c}{ Genotype of Mob1a } \\
& Total & $+/+$ & $\Delta /+$ & $\Delta / \Delta$ & Absorbed \\
Viable & 108 & 39 & 69 & 0 & \\
E16.5 & 3 & 1 & 2 & 0 & \\
E14.5 & 15 & 4 & 11 & 0 & \\
E10.5 & 19 & 8 & 9 & 0 & 2 \\
E9.5 & 27 & 11 & 13 & 0 & 3 \\
E7.5 & 19 & 3 & 12 & 0 & 4 \\
E6.5 & 47 & 11 & 23 & 0 & 13 \\
E3.5 & 33 & 9 & 16 & 0 & 0 \\
\hline
\end{tabular}


A
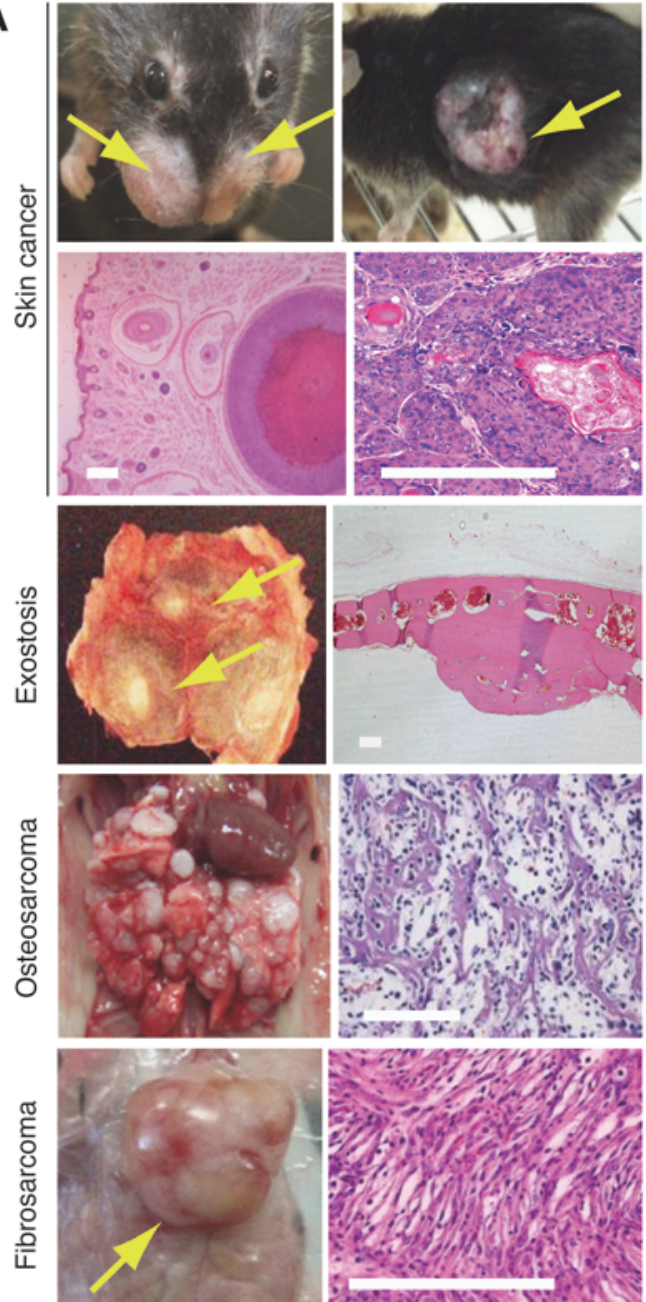
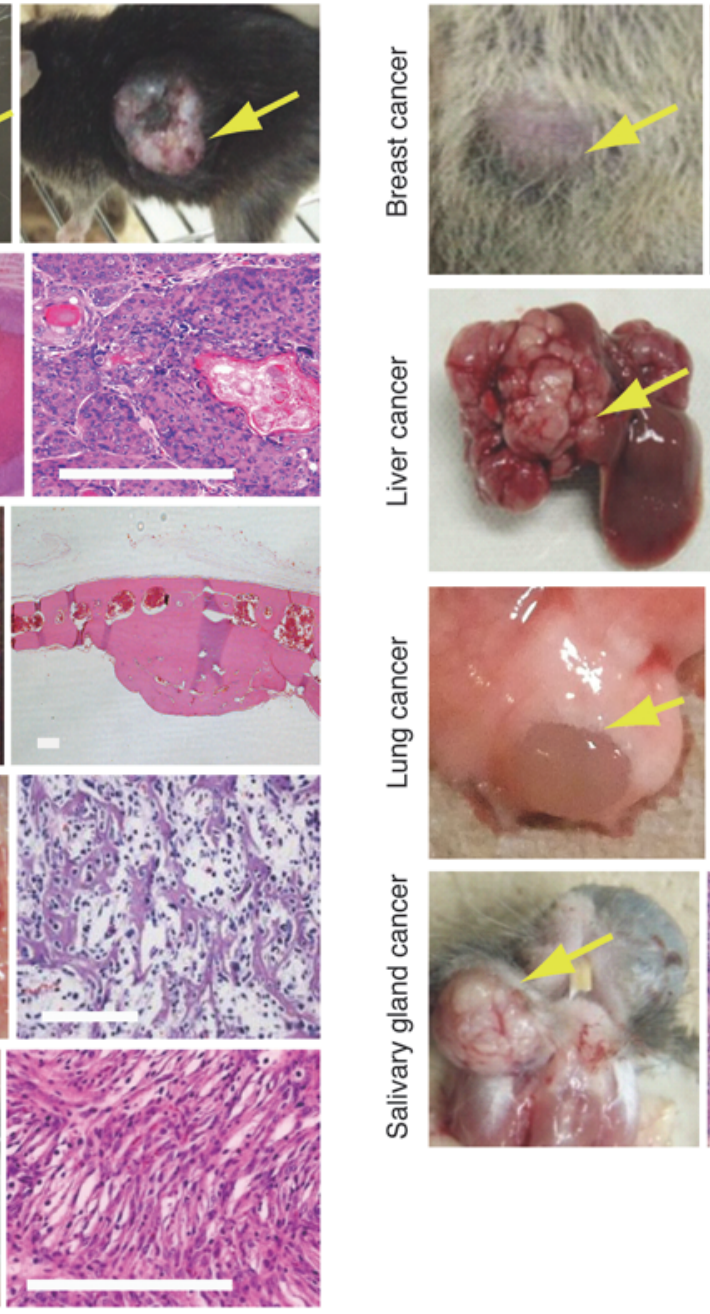
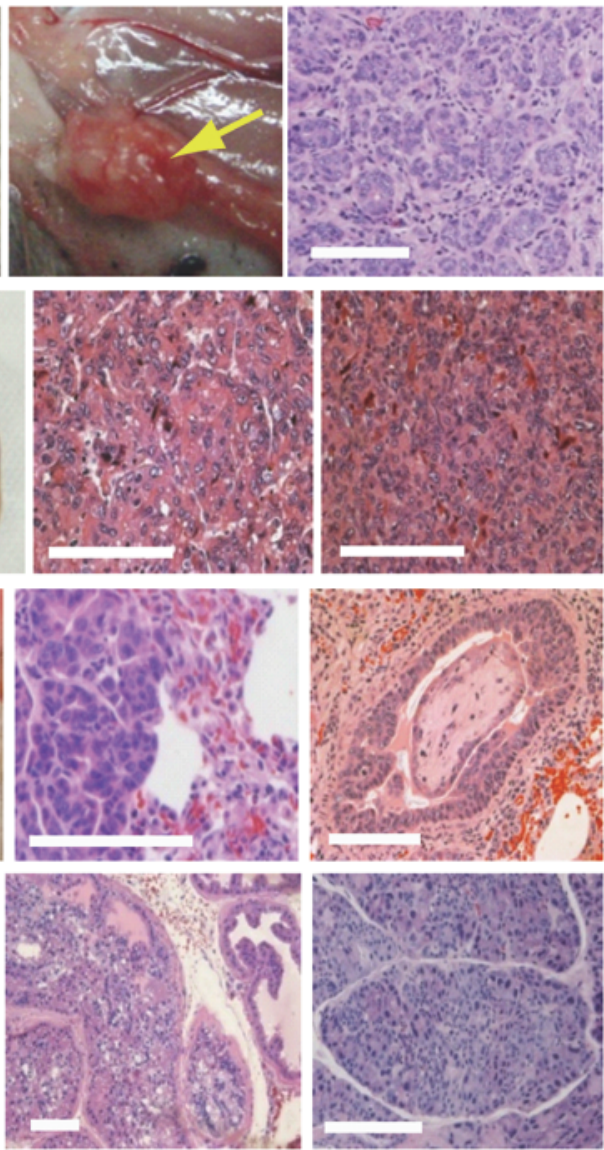

B

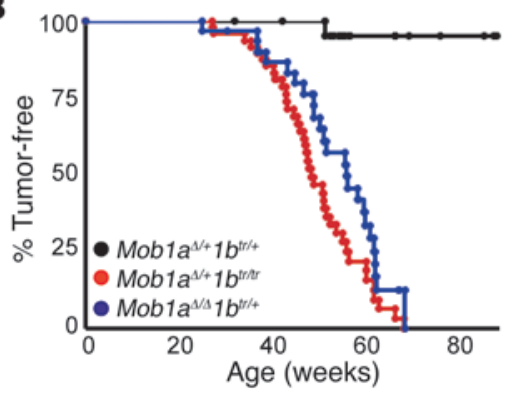

C
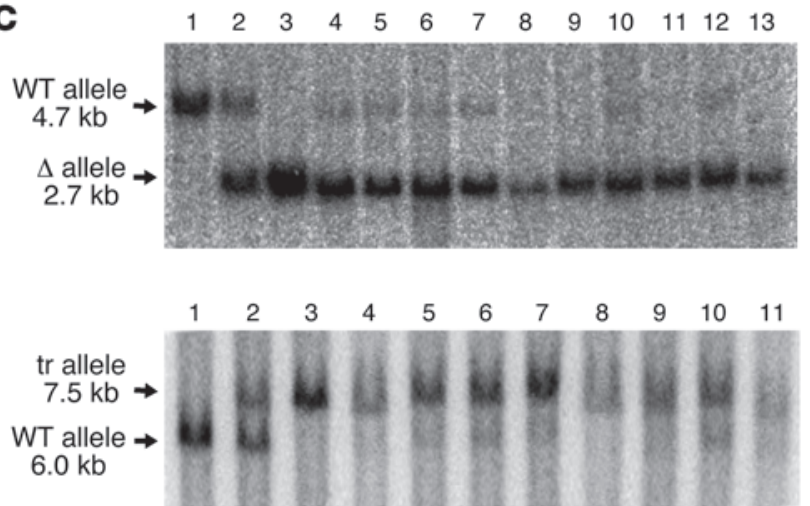

\section{Figure 2}

Loss of Mob1a/1b promotes tumorigenesis. (A and B) Tumor types arising in Mob1a $a^{\Delta / \Delta} 1 b^{t r /+}$ or Mob1 $a^{\Delta /+} 1 b^{t r / t r}$ mice. (A) Macroscopic or microscopic photographs of the indicated gross or H\&E-stained tumors in mutant mice. Yellow arrows, tumor masses. Scale bars: $100 \mu \mathrm{m}$. (B) KaplanMeier analysis of tumor onset and survival for the Mob1 $a^{\Delta / \Delta} 1 b^{t r /+}(n=31)$, Mob1 $a^{\Delta+1} b^{t r / t r}(n=48)$, and control Mob1a $a^{\Delta /+1} b^{t r /+}(n=22)$ mice in $\mathbf{A}$. (C) Southern blots of tumor DNA showing loss of the WT Mob1 allele. Top (hybridized to probe A of Supplemental Figure 1A): lanes 1-3, control thymic DNA from Mob1 $\mathrm{a}^{+/+}$, Mob1a $\mathrm{a}^{\Delta /+}$, and Mob1a $\mathrm{a}^{\Delta / \Delta}$ mice, respectively; lanes 4-5, osteosarcomas; 6-7, fibrosarcomas; 8-9, skin cancers; 10-11, breast cancers; $12-13$, salivary gland cancers of Mob1 $a^{\Delta /+} 1 b^{t r / t r}$ mice. Bottom (probe C): $1-3$, control tail DNA of Mob1 $b^{+/+}, M o b 1 b^{t r /+}$, and Mob1 $b^{\text {tr/tr }}$ mice, respectively; 4-5, osteosarcomas; 6-7, fibromyosarcomas; 8-9, skin cancers; $10-11$, liver cancers of Mob1 $a^{\Delta / \Delta} 1 b^{t r /+}$ mice. Results shown are representative of at least 3 independent trials and at least 3 mice/group. Data are presented as the mean \pm SEM, and $P$ values were determined using the 2-tailed Student's $t$ test. 
Table 2

Total tumor numbers (incidence) for the mice in Figure $2 \mathrm{~A}$

\begin{tabular}{lccc}
\hline & Mob1a $^{\Delta /+} \mathbf{1}^{\text {tr/tr }}$ & $\boldsymbol{M o b 1 a}^{\Delta / \Delta} \boldsymbol{1}^{\text {tr/+ }}$ & Overall \\
Skin cancer & $23(100 \%)$ & $14(100 \%)$ & $37(100 \%)$ \\
Exostosis & $20(87 \%)$ & $14(100 \%)$ & $34(92 \%)$ \\
Osteosarcoma & $7(30 \%)$ & $2(14 \%)$ & $9(24 \%)$ \\
Fibrosarcoma & $6(26 \%)$ & $2(14 \%)$ & $8(22 \%)$ \\
Liver cancer & $0(0 \%)$ & $7(50 \%)$ & $7(19 \%)$ \\
Breast cancer & $5(22 \%)$ & $1(7 \%)$ & $6(16 \%)$ \\
Lung cancer & $0(0 \%)$ & $2(14 \%)$ & $2(5 \%)$ \\
Salivary gland cancer & $1(4 \%)$ & $1(7 \%)$ & $2(5 \%)$ \\
\hline
\end{tabular}

tosis in calvaria occurred in $92 \%$ of animals with just 1 WT Mob1 allele as well as extraskeletal osteosarcomas (24\%), subcutaneous fibrosarcomas (or myofibrosarcomas) (22\%), breast cancers (16\%), lung cancers (5\%), and salivary gland cancers (5\%). Liver cancers were found only in Mob1 $a^{\Delta / \Delta} 1 b^{t r /+}$ mutants (50\%). Interestingly, none of our Mob1-deficient mice developed colon tumors or melanomas, unlike MOB1A-deficient humans $(17,33)$. Nevertheless, MOB1 is a powerful tumor suppressor in multiple tissues in vivo.

Keratinocyte-specific Mob1a/1b-deficient mice show gross skin abnormalities and die during lactation. Because the dominant tumor in Mob1 $a^{\Delta /+} 1 b^{t r / t r}$ and Mob1 $a^{\Delta / \Delta} 1 b^{t r /+}$ mice arose in the skin, we generated tamoxifen-inducible, keratinocyte-specific Mob1a/1b doublehomozygous mutant mice (Krt14CreERMob1 $a^{f l / f l} 1 b^{t r / t r}$ ). The Keratin-14 (Krt14) promotor directs gene expression in the basal layer of epidermal and follicular keratinocytes, including stem cells in the HF bulge. Thus, tamoxifen-induced, Krt14-controlled Cremediated deletion of a floxed gene disrupts expression of that gene throughout the IFE and HFs (35). When we administered tamoxifen to Krt14CreERMob1aflfl $1 b^{t r / t r}$ mice at P1, we created doublehomozygous conditional mutant mice $[k \mathrm{DKO}(\mathrm{P} 1)]$ that initially appeared healthy. Single-mutant mice (Krt14CreERMob1 $a^{\text {fl/fl }} 1 b^{t r / t r}$ ) without tamoxifen or Krt14CreERMobla $a^{f / f l} 1 b^{+/+}$mice with tamoxifen were indistinguishable from the WT in gross appearance, histology, and survival (data not shown), allowing us to choose Krt14CreERMob1 al/fl $1 b^{t r / t r}$ mice without tamoxifen as controls. Southern blotting confirmed that tamoxifen disrupted the Mob1 $a^{\text {flox }}$ allele in almost all keratinocytes of $k \mathrm{DKO}(\mathrm{P} 1)$ mice, leaving the Mob1 $a^{\Delta}$ allele (Supplemental Figure 3A). In addition, immunostaining using an $\mathrm{Ab}$ recognizing both MOB1A and MOB1B (Supplemental Figure 3B) confirmed that the MOB1A and MOB1B proteins are expressed both in IFE cells (especially in the granular layer) and in HF cells (especially in matrix cells and outer root sheath cells) in normal mice and humans (Supplemental Figure 3, C and D). In contrast, MOB1A and MOB1B proteins were not detected in the epidermis of $k \mathrm{DKO}(\mathrm{P} 1)$ mice (Supplemental Figure 3C).

By 13 days of age, $k \mathrm{DKO}(\mathrm{P} 1)$ mice were significantly smaller than control littermates and readily identified by their "wrinkledbear" facial skin (Figure 3A) and ruffled hair (Figure 3A). These mutants' front paws (Figure 3A), ears, and lips were hyperplastic and enlarged. All $k \mathrm{DKO}(\mathrm{P} 1)$ mice died of malnutrition within 10-30 days of birth (Figure 3), likely due to dysphagia caused by hyperplasia of the oral cavity epithelium.

We next analyzed the architecture of $k \mathrm{DKO}(\mathrm{P} 1)$ skin at various time points after birth. In WT mice, dividing keratinocytes are restricted to the basal epidermal layer. As these cells exit the cell cycle, they differentiate to form the spinous and granular skin layers as well as the dead, enucleated stratum corneum layers. The anagen phase of hair development thickens the skin until day 17, when the first catagen phase is triggered and skin thickness regresses until day 19. Hair remodeling then begins its lifelong cycle of spontaneous regrowth and regression (36). We saw this pattern clearly in our biopsy series from control mice (Supplemental Figure 4). However, the IFE and HFs of $k \mathrm{DKO}(\mathrm{P} 1)$ mice showed hyperplastic multilayered epithelium at day 16 (Figure 3B), and impaired epidermal regression during catagen (Supplemental Figure 4; day 19). In addition, parakeratosis (reduced enucleation) was evident in the stratum corneum layer of $k \mathrm{DKO}(\mathrm{P} 1)$ mice (Figure 3C).

To investigate keratinocyte differentiation, we used immunostaining to detect keratin markers in the fat pad epidermis, which allows easy analysis of epidermal layers. Keratin- $15^{+}\left(\right.$KRT15 $\left.{ }^{+}\right)$ cells, the most primitive in IFE, normally form a single basal layer attached to the IFE basement membrane (BM). This configuration was clearly visible in our controls, whereas the KRT $15^{+}$cells of $k \mathrm{DKO}(\mathrm{P} 1)$ mice, although nearly normal in number, were scattered inside the IFE without attachment to the BM (Figure 3D). In control mice, $\mathrm{KRT}_{14}{ }^{+}$and $\mathrm{KRT}^{+}$cells were present in 1 or 2 IFE layers (as expected), but appeared in multiple layers in $k \mathrm{DKO}(\mathrm{P} 1)$ mice (Figure 3D). Moreover, there were increased numbers of irregularly organized KRT10 $0^{+}$cells in the suprabasal layers of $k \mathrm{DKO}(\mathrm{P} 1)$ epidermis (Figure 3D). Filaggrin ${ }^{+}$cells, the most highly differentiated keratinocytes, were present in $k \mathrm{DKO}(\mathrm{P} 1)$ epidermis but at a reduced percentage (Figure 3D). Similar IFE alterations were observed in the back skin of $k \mathrm{DKO}(\mathrm{P} 1)$ mice (Figure $3 \mathrm{E}$ and Supplemental Figure 5). KRT17 ${ }^{+}$outer root sheath cells were significantly increased in back skin HFs of $k \mathrm{DKO}(\mathrm{P} 1)$ mice, and Trichohyalin ${ }^{+}$inner root sheath cells were elevated slightly (Figure $3 \mathrm{E})$. Thus, the phenotype of $k \mathrm{DKO}(\mathrm{P} 1)$ mice may be largely due to abnormal expansion of immature stem/progenitor skin cells.

To try to overcome the early lethality of $k \mathrm{DKO}(\mathrm{P} 1)$ mice, we waited until $k \mathrm{DKO}$ mice reached 28 days of age before treating them with tamoxifen for 7 days. However, like $k \mathrm{DKO}(\mathrm{P} 1)$ mice, all of these $k \mathrm{DKO}(\mathrm{P} 28)$ mice showed keratinocyte hyperplasia (Figure 3F) and died within 15-55 days after treatment (Supplemental Figure 6). Thus, MOB1 deficiency in epidermal cells severely disrupts normal IFE and HF development and homeostasis.

Tumorigenic anomalies in Mob1a/1b double-homozygous mutant keratinocytes. To determine whether loss of MOB1 conferred tumorigenic properties on keratinocytes, we first immunostained $k \mathrm{DKO}(\mathrm{P} 1)$ skin with anti-Ki67 $\mathrm{Ab}$ and found increased numbers of $\mathrm{Ki}^{+}{ }^{+}$ proliferating epithelial cells (Figure 4A). In control mice at P13, a few $\mathrm{Ki} 67^{+}$cells were located among basal IFE cells and outer root sheath cells, with more scattered among HF matrix cells. However, the incidence of $\mathrm{Ki}^{+} 7^{+}$cells in $k \mathrm{DKO}(\mathrm{P} 1)$ skin at $\mathrm{P} 13$ was 1.5 times higher than in controls, and $\mathrm{Ki} 67^{+}$cells were also found in the suprabasal layers normally quiescent in control epidermis. In addition, TUNEL-positive apoptotic cells were reduced in $k \mathrm{DKO}(\mathrm{P} 1)$ epidermis (Figure 4B). Thus, increased proliferation and repressed apoptosis may contribute to $k \mathrm{DKO}(\mathrm{P} 1)$ epidermal hyperplasia. Cell-plating studies revealed that the saturation density of $k \mathrm{DKO}(\mathrm{P} 1)$ keratinocytes was also increased (Figure 4C). Because the cell size (forward scatter) of control and mutant keratinocytes showed no difference by FACS analysis (data not shown), the increased saturation density of the mutant suggests impaired contact inhibition. Histological analysis confirmed that the mutant basal epidermal layer showed significantly increased cell density 

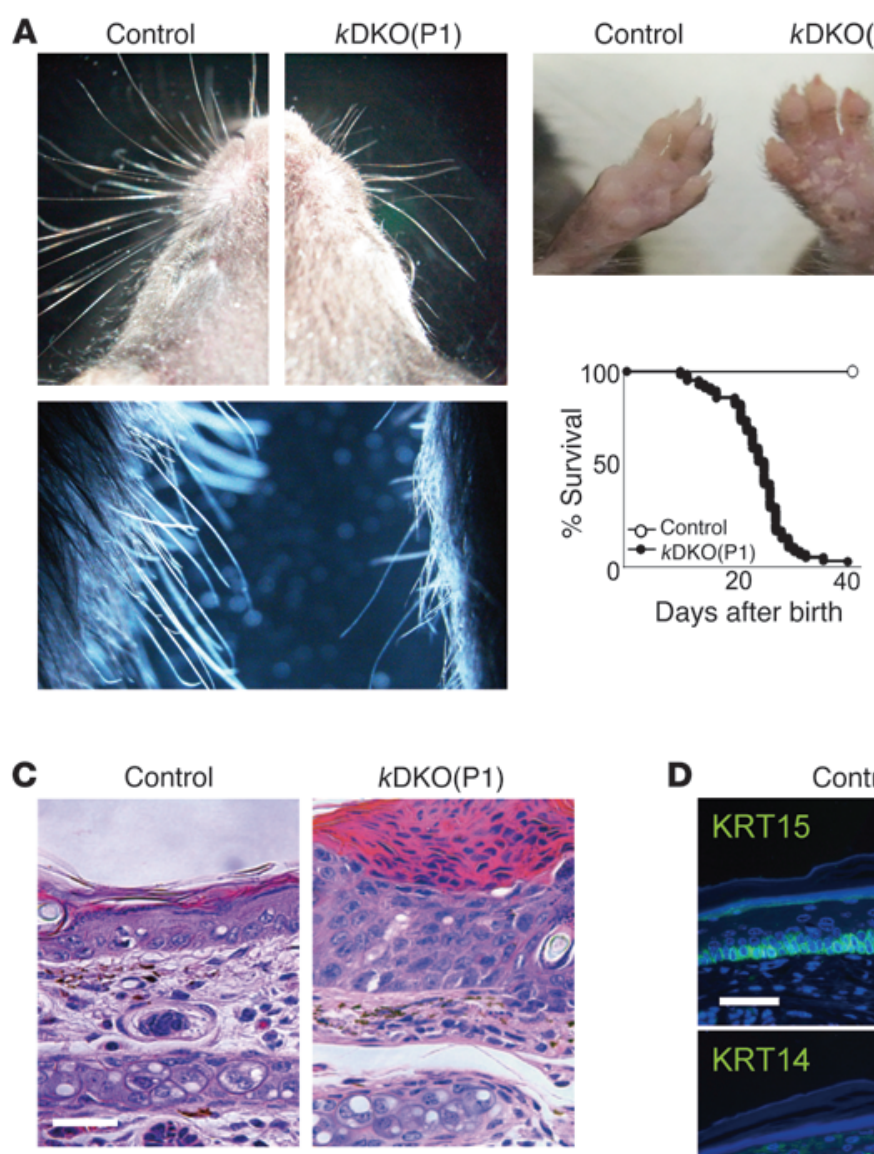

E
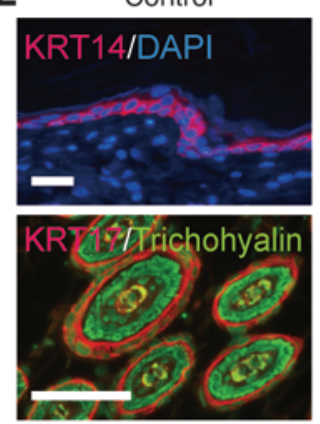

F Control

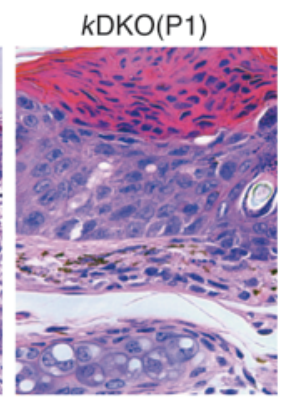

kDKO(P1)
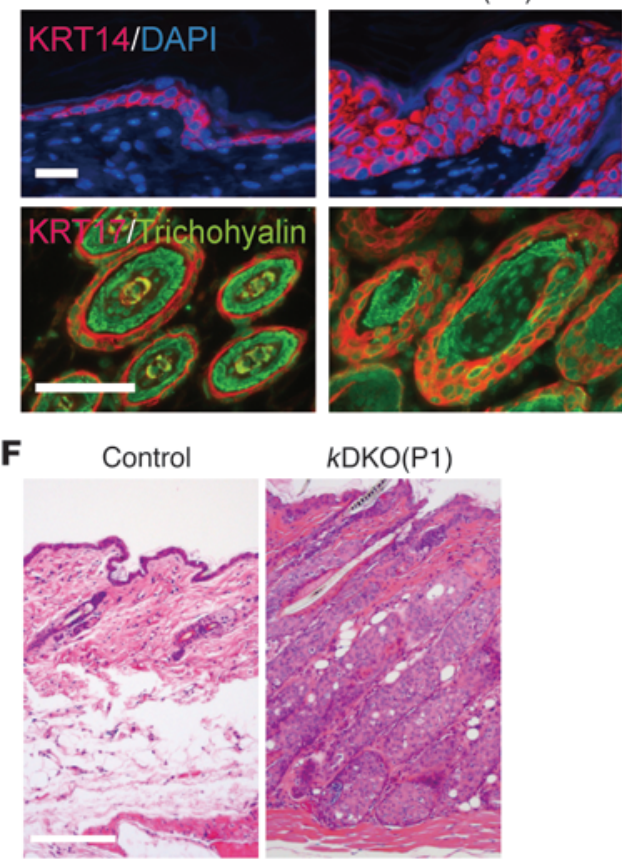

D
B
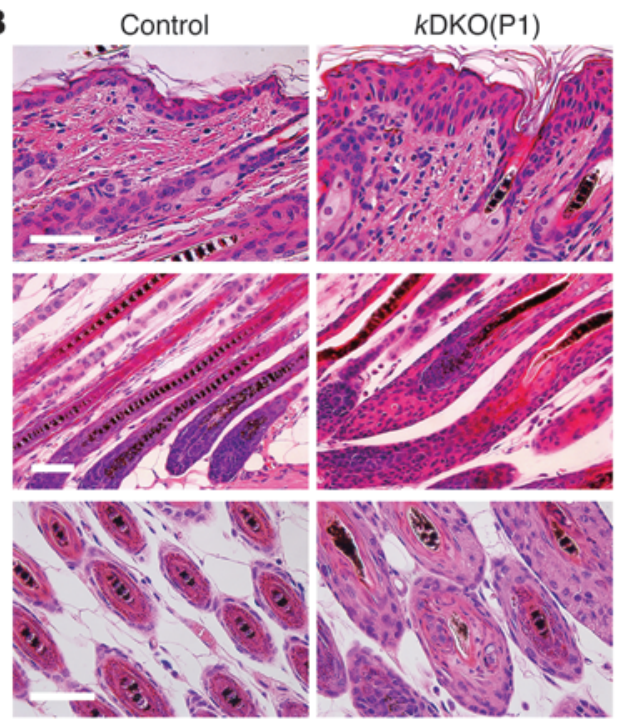

Control

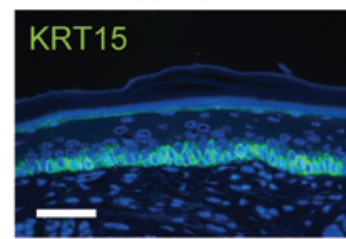

$k \mathrm{DKO}(\mathrm{P} 1)$
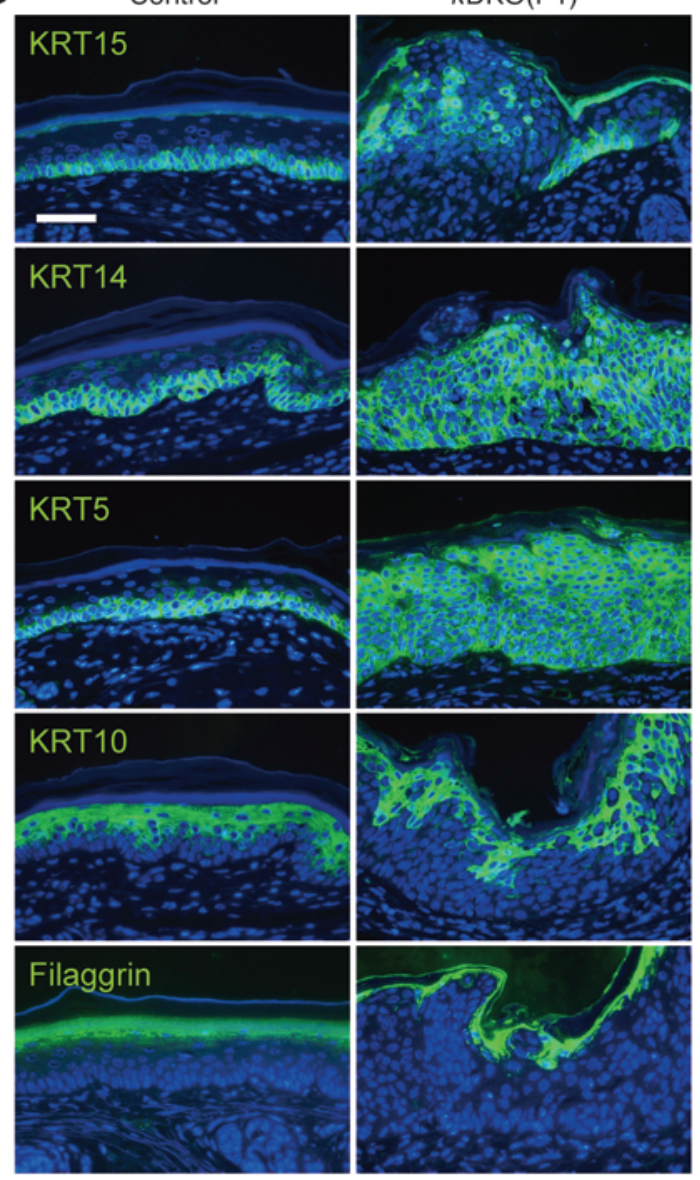


\section{Figure 3}

Characterization of keratinocyte-specific Mob1a/1b double-homozygous mutant mice. (A-C) Features of 16-day-old Krt14CreERMob1a $a^{f / f} 1 b^{t r / t r}$ mice that were originally left untreated (control) or treated with tamoxifen $(k D K O)$ at $\mathrm{P} 1$. (A) $k \mathrm{DKO}(\mathrm{P} 1)$ mice have a wrinkled-bear facial appearance (top left), ruffled, shaggy body hair (bottom left), abnormally large front paws (top right), and decreased survival (bottom right). (B) H\&Estained longitudinal and transverse sections of the back skin of the mice in A. $k D K O(P 1)$ mice show hyperplasia of IFE and HF. Scale bars: 50 $\mu \mathrm{m}$. (C) $k \mathrm{DKO}(\mathrm{P} 1)$ mice show parakeratosis (reduced enucleation) in stratum corneum layers. Scale bar: $20 \mu \mathrm{m}$. (D) Immunohistochemical analyses of fat pad epidermis of the mice in A using Alexa Fluor 488tagged Abs recognizing the indicated differentiation markers corresponding to a specific skin layer. DAPI, nuclei. Scale bar: $50 \mu \mathrm{m}$. (E) Immunohistochemical analyses of back skin of the mice in A using Abs recognizing the indicated skin layer markers. For top panels, cells were stained with Alexa Fluor 568-tagged anti-KRT14 Ab. DAPI, nuclei. Scale bars: $20 \mu \mathrm{m}$. For bottom panels, cells were stained with Alexa Fluor 488-tagged anti-Trichohyalin Ab and Fluor 568-tagged anti-KRT17 Ab. Scale bar: $50 \mu \mathrm{m}$. (F) H\&E-stained longitudinal sections of skin from 42-day-old Krt14CreERMob1a $a^{f / f t} 1 \mathrm{~b}^{\text {tr/tr }}$ mice that were left untreated (control) or treated with tamoxifen (kDKO) at P28. The $k D K O(P 28)$ mutant shows hyperplasia of IFE and HFs. Scale bar: $200 \mu \mathrm{m}$. Results shown are representative of at least 3 independent trials and at least 3 mice/group. Data are presented as the mean \pm SEM.

(control: $7.6 \pm 0.36$ nuclei per $50 \mu \mathrm{m} \mathrm{BM}$; mutant: $8.9 \pm 0.38$ nuclei per $50 \mu \mathrm{m} \mathrm{BM} ; n=5 ; P<0.05)$. Morphologically, mutant basal cells were elongated and columnar rather than round like control basal cells (Figure 4D), also indicating defective contact inhibition.

Because yeast $M O B 1$ regulates mitotic exit and cytokinesis (24-29), we speculated that mammalian MOB1 might affect processes that support cell division. Indirect immunofluorescence analysis of control and $k \mathrm{DKO}(\mathrm{P} 1)$ keratinocytes using anti- $\gamma$-tubulin and anti- $\alpha$-tubulin Abs revealed an increase in centrosome number $(>2$ centrosomes/cell) (Figure 4E). Aberrant centrosome numbers are associated with multipolar spindle formation that impairs chromosome segregation. Indeed, a greater percentage of $k \mathrm{DKO}(\mathrm{P} 1)$ keratinocytes exhibited multi-polar spindles (Figure 4F). Micronuclei, which are cytoplasmic chromosomal fragments generated by aberrant mitosis, were also increased in $k \mathrm{DKO}(\mathrm{P} 1)$ keratinocytes (Figure 4G). Similar defects were observed in Mob1a and Mob1b double-homozygous mutant embryonic fibroblasts (MEFs) (Supplemental Figure 7, A-C).

To determine which mitotic stage was altered in $k \mathrm{DKO}(\mathrm{P} 1)$ keratinocytes, we divided the mitotic process into 4 parts based on cell morphology: part I, cells progressing from the round morphology of a resting cell to chromosome alignment (corresponding to prometaphase); part II, from chromosome alignment to ingression of the cleavage furrow (metaphase/late anaphase); part III, from ingression to furrow completion (late anaphase/telophase); and part IV, from furrow completion to cell division (cytokinesis). We used timelapse video microscopy to measure the duration of each of parts I-IV in control and $k \mathrm{DKO}(\mathrm{P} 1)$ keratinocytes. Part IV was significantly accelerated in MOB1-deficient cells compared with controls, while part I was slightly faster in the mutant (Supplemental Figure 8, A and B). No significant differences were detected for parts II and III. Thus, loss of MOB1 accelerates a cell's exit from mitosis.

We noted that $k \mathrm{DKO}(\mathrm{P} 1)$ mice had an apparent increase in the number of skin progenitors in the IFE (Figure 3D). We therefore performed RT-PCR to evaluate stem/progenitor cells in the HFs.
$\mathrm{CD} 4^{+}$and $\mathrm{LGR}^{+}$cells are the most immature HF stem cells located in the bulge and isthmus. We found that Cd34 and Lgr6 mRNAs were normal or reduced in $k \mathrm{DKO}(\mathrm{P} 1)$ keratinocytes, but that markers of activated stem/progenitor cells, such as Sox 9 and $\operatorname{Lgr} 5$, were elevated (Figure $4 \mathrm{H}$ ). These findings were confirmed by FACS analysis and immunohistochemistry (Figure 4H). However, despite their normal numbers, CD $34^{+}$cells in mutant HFs could not localize to the proper bulge area (Supplemental Figure 9). This result mirrors the failure of HF stem cells to localize to the basal cell layer in MOB1-deficient IFE and HFs (Figure 3D). In addition, SOX9 $9^{+}$cells (which are normally located in the bulge at P21) were markedly increased among $k \mathrm{DKO}(\mathrm{P} 1)$ keratinocytes and scattered throughout the HFs (Figure 4H). To determine how MOB1 inactivation affected HF stem cell self renewal, we quantified the ability of the total $k \mathrm{DKO}(\mathrm{P} 1)$ keratinocyte population to form colonies in culture. A lack of MOB1 induced a 2.1-fold increase in colonyforming efficiency (Figure 4I). When these primary colonies were replated to test their ability to form secondary colonies, a 3.0-fold increase in secondary colony-forming efficiency was observed in the absence of MOB1 (Figure 4I). Thus, MOB1 inactivation enhances the self renewal of activated stem/progenitor keratinocytes. This property, along with hyperproliferation, apoptotic resistance, impaired contact inhibition, increased centrosome number, and accelerated mitotic exit, may account for the frequent cancers observed in Mob1-deficient mice.

MOB1-mediated regulation of the LATS1/2-YAP1 pathway controls skin bomeostasis. To characterize the mechanisms driving the $k \mathrm{DKO}(\mathrm{P} 1)$ phenotype, we investigated the effects of MOB1 loss on signaling mediated by YAP1 and its upstream kinases LATS1/2. In vitro, MOB1 is important for the full activation of LATS kinases (37), and activated LATS1/2 phosphorylate YAP1 and inhibit its nuclear localization (38). Using immunohistochemistry, we found that, in control mice, YAP1 was localized in the nuclei of most outer root sheath cells, in some HF matrix cells, and in some IFE basal cells (Figure 5A). However, $k \mathrm{DKO}(\mathrm{P} 1)$ mice exhibited not only greater numbers of nuclear YAP1 ${ }^{+}$cells in these sites but also some nuclear $\mathrm{YAP}^{+}{ }^{+}$cells in the upper IFE layer (Figure 5A). Immunoblotting of keratinocytes confirmed that the phosphorylation of YAP1 on Ser127 was reduced, but that total YAP1 was increased in the absence of MOB1 (Figure 5B), especially in the nucleus (Figure $5 \mathrm{C}$ ). When we cultured keratinocytes under high cell density conditions, YAP1 was inactivated and localized in the cytoplasm in control cells (Figure 5D). In contrast, in $k \mathrm{DKO}(\mathrm{P} 1)$ keratinocytes, YAP1 remained activated and positioned in the nucleus even at high-cell density (Figure 5D). Thus, MOB1 is required for densityinduced subcellular localization of YAP1.

We next investigated LATS $1 / 2$ phosphorylation in control and mutant keratinocytes. Immunoblotting demonstrated that, in the absence of any stimulation, levels of phospho-LATS1(T1079) and phospho-LATS2(T1041) were below the assay detection limit even in control keratinocytes (Figure 5E). We then stimulated control and $k \mathrm{DKO}(\mathrm{P} 1)$ keratinocytes with okadaic acid (OA), which activates the MST kinases acting upstream of LATS1/2 (39). While OAstimulated control keratinocytes showed vigorous phosphorylation of LATS1/2, OA-stimulated kDKO(P1) keratinocytes did not. Strikingly, total LATS1/2 protein levels were also reduced in the mutant. This inhibition of LATS1/2 phosphorylation was not due solely to the reduction in total LATS1/2 proteins because levels of phosphoLATS1/2 were also markedly decreased when total LATS1/2 protein levels in each sample were adjusted to equality prior to electropho- 
A

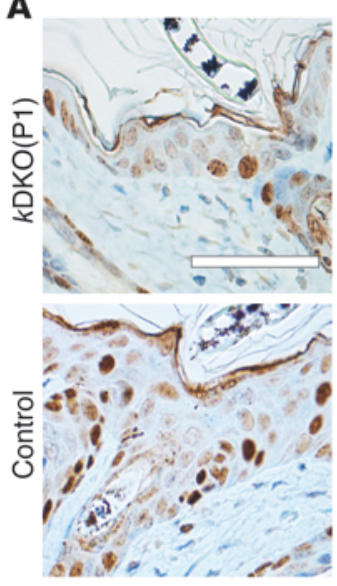

C

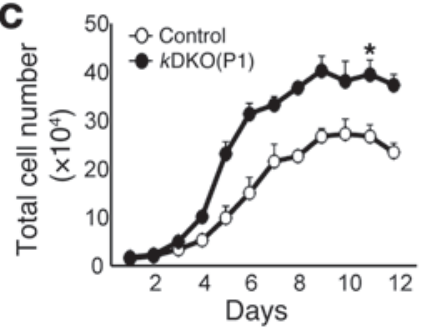

E Control
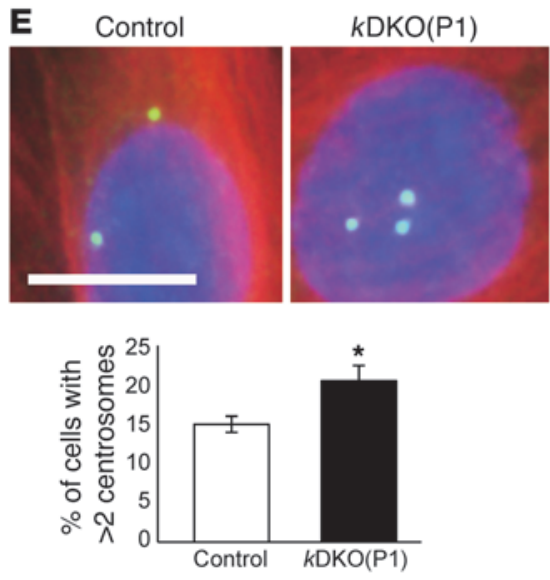

H $\%$ of control
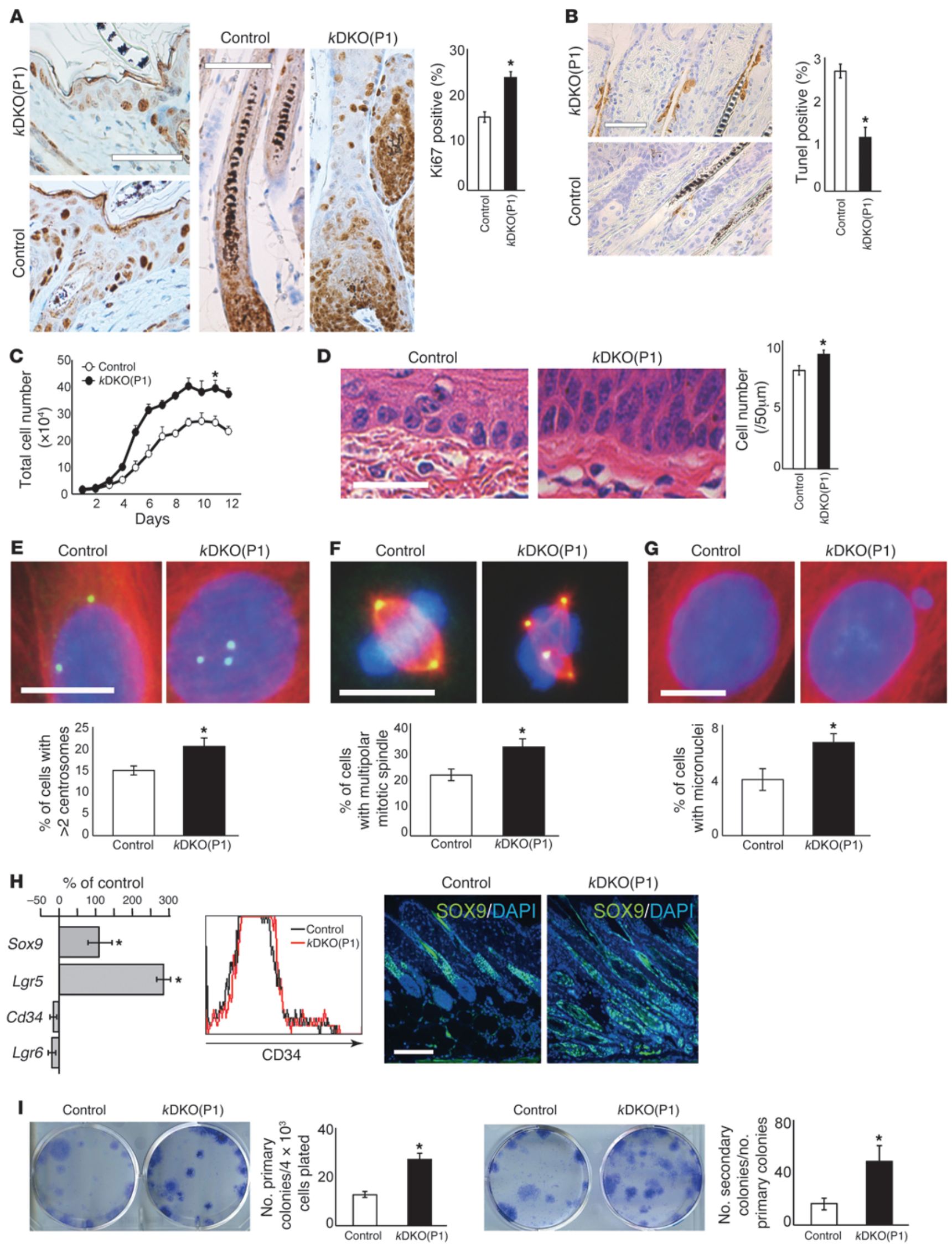

Control

$k \mathrm{DKO}(\mathrm{P} 1)$
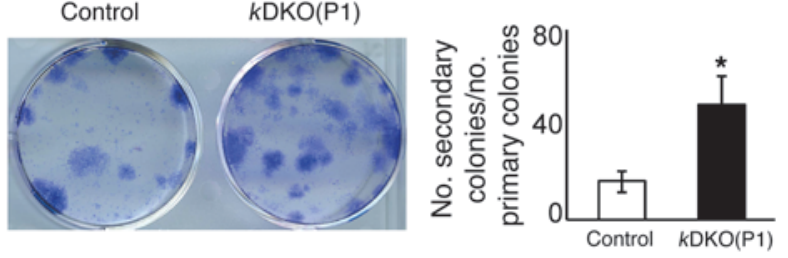


\section{Figure 4}

Tumorigenic anomalies in Mob1a/1b double-homozygous mutant keratinocytes. (A) Anti-Ki67 immunostaining of IFE (left) and HF (middle) of control and $k D K O(P 1)$ mice at P13. Scale bars: $50 \mu \mathrm{m}$. Quantitation of Ki67 ${ }^{+}$cells (right); ${ }^{\star} P<0.01$. (B) Histology (left) and quantitation (right) of TUNEL-stained cells in epidermis from control and $k D K O(P 1)$ mice at P16. Scale bar: $50 \mu \mathrm{m} ;{ }^{*} P<0.01$. (C) Keratinocytes from control and $k D K O(P 1)$ mice at P4 were cultured for the indicated number of days, and total cell numbers were counted. $k D K O(P 1)$ keratinocytes achieved higher saturation plating density; ${ }^{\star} P<0.01$. (D) Left: H\&E-stained epidermal basal layer of control and $k D K O(P 1)$ mice at P19. Scale bar: $20 \mu \mathrm{m}$. Right: quantitation (cell number/50 $\mu \mathrm{m} \mathrm{BM);} n=5 /$ group; ${ }^{\star} P<0.05$. (E-G) Immunostaining to detect $\gamma$-Tubulin (green) and $\alpha$-Tubulin (red) in control and $k D K O(P 1)$ keratinocytes. DAPI, nuclei. Mutant keratinocytes showed excess centrosomes (E), multi-polar spindles $(\mathbf{F})$, and micronuclei $(\mathbf{G})$. Scale bars: $20 \mu \mathrm{m}$; ${ }^{*} P<0.05$. (H) Identification of keratinocyte stem cells in HFs of control and $k \mathrm{DKO}(\mathrm{P} 1)$ mice at P19 ( $n=4$ /group) using quantitative RT-PCR (left), ${ }^{*} P<0.01$; flow cytometry (middle) to detect CD34; immunostaining (right) to detect SOX9. Scale bar: $100 \mu \mathrm{m}$. (I) Freshly isolated control and $k \mathrm{DKO}(\mathrm{P} 1)$ keratinocytes were plated to generate primary colonies (left) and secondary colonies (right). Giemsa staining (top) and colony counts (bottom) were performed on day 14 after plating; $n=4 /$ group; ${ }^{*} P<0.02$. Results shown are representative of at least 3 independent trials and at least 3 mice/group. Data are presented as the mean \pm SEM, and $P$ values were determined using the 2-tailed Student's $t$ test.

resis (Figure 5E). These data show that MOB1 regulates LATS1/2YAP1 signaling essential for mammalian skin homeostasis.

$M O B 1$ is involved in the onset of malignant outer root sheath tumors resembling trichilemmal carcinomas. Microscopic analysis of the skin tumors of Mob1 $a^{\Delta / \Delta} 1 b^{t r /+}$ and Mob1 $a^{\Delta /+} 1 b^{t r / t r}$ mice revealed lesions consisting of invasive epithelial lobules with atypical cytology, an increased number of mitotic cells, and horn cysts (Figure 6A). Trichilemmal keratinization and continuity with the follicular epithelium (Figure 6A) were focally evident. Because these features are characteristic of trichilemmal carcinomas of outer root sheath origin, we immunostained these tumors with Abs recognizing KRT17, KRT5, KRT15, KRT10, Trichohyalin, or AE13. All tumors were positive for KRT17 (outer root sheath marker), KRT5 (basal cells and outer root sheath), and KRT15 (basal cells, outer root sheath, and bulge), but negative for KRT10 (upper basal IFE) and Trichohyalin (inner root sheath) (Figure 6B). Some tumors were also positive for AE13 (hair shaft marker), confirming that these malignancies were derived from the HF outer root sheath. Thus, deficiency for the Hippo signaling element MOB1 in mice leads to tumors with the features of trichilemmal carcinomas.

In humans, the most common HF-derived carcinomas are nodular BCCs, which are driven by hyperactivated SHH signaling (5). However, immunoblotting of our MOB1-deficient keratinocytes showed that neither GLI2 (downstream effector in the SHH pathway) nor the LEF1 or HES1 proteins important for HF morphogenesis were elevated (Figure 6C). In addition, while AKT was not activated in the mutant keratinocytes, phospho-ERK was increased (Figure 6C). To rule out the involvement of the $\mathrm{SHH}$ pathway in tumorigenesis linked to MOB1 deficiency, we immunostained control and mutant keratinocytes with Abs validated to detect GLI2 or GLI1 (Supplemental Figure 10, A and B) in patterns consistent with a previous report (40). However, when we immunostained control and $k \mathrm{DKO}(\mathrm{P} 1)$ keratinocytes with these Abs, no differences were detected (Figure 6D and Supplemental Figure 10C). Furthermore, GLI2 levels in 4 trichilemmal carcino- ma samples from $k \mathrm{DKO}$ (P1) mice were not obviously increased compared with GLI2 levels in cultured control keratinocytes (Figure $6 \mathrm{E}$ ). Thus, the SHH pathway involved in nodular BCCs does not appear to be driving the development of the trichilemmal carcinomas arising in the absence of MOB1A/1B.

In humans, trichilemmal carcinomas are very rare and composed mainly of basaloid and squamous cells, with some clear cells (Figure $6 \mathrm{~F}$ ). We performed immunostaining to detect YAP1 in samples of 14 human trichilemmal carcinomas with accompanying noncancerous epidermis. We found that, in noncancerous skin, YAP1 was expressed in the nuclei of IFE basal cells and HF outer root sheath cells (Figure 6G). Strikingly, a significant proportion $(11 / 14,79 \%)$ of trichilemmal carcinomas showed an increase in total YAP $1^{+}$cells (Figure 6G and Table 3 ). Almost as many (10/14, $71 \%)$ trichilemmal carcinomas exhibited elevated nuclear YAP1 within the tumor mass (Figure $6 \mathrm{G}$ and Table 3 ). In addition, when immunostained to detect MOB1A/1B, half of trichilemmal carcinomas examined $(5 / 10,50 \%)$ showed decreased MOB1A/1B expression (Figure 6G and Table 3). Moreover, 10/10 (100\%) of these cases in which MOB1 was decreased displayed elevated nuclear YAP1 within the tumor. These data support our hypothesis that it is the mammalian Hippo pathway, rather than the SHH pathway, that is involved in the onset of non-BCC follicular cancers such as trichilemmal carcinomas.

\section{Discussion}

We have demonstrated that mice completely deficient for Mob1 have the most severe phenotype among strains lacking a Hippo signaling component. Mob1a/1b double-homozygous-null mutant mice die at gastrulation, much earlier than mice lacking Mst1/2 (15), Lats2 (21), Lats1 (14), Sav1 (20), or Nf2 (19). Our data further indicate that MOB1 is essential for embryogenesis and that functions of MOB1A and MOB1B overlap. In addition, the tumor spectrum observed in heterozygous Mob1-deficient mice is the broadest among mutants lacking Hippo components. These findings suggest both that MOB1 is the key molecule in the Hippo signaling pathway and that MOB1 may have molecular target(s) other than the Hippo pathway. This notion is consistent with the reduced expression or mutation of MOB1 frequently observed in a variety of human cancers $(17,33,34)$, and with MOB1's reported binding to a range of molecules, including TSSC1, NUP98, HDAC3, and DIPA (CCDD85B) $(41,42)$. Our results also imply that MOB1A may be more important than MOB1B, at least for embryogenesis and liver homeostasis, because Mob1b heterozygotes lacking Mob1a show partial embryonic lethality (Supplemental Table 1) and develop liver cancers (Table 2), whereas Mob1a heterozygotes lacking Mob1b are all viable and free of liver tumors.

The lethality of Mob1-deficient mice may stem from their failure to form primitive endoderm. The endodermal markers Pdgfra and Gata4 were markedly reduced in Mob1-deficient cells, whereas the primitive ectoderm markers Nanog, Sox2, Fgf5, and Pax6 were normal. Our mutant showed abnormal YAP1 activation, and activated YAP1 normally activates the transcription factor TEAD2. TEAD2 regulates primitive endoderm-specific genes such that sustained inhibition of TEAD2 enhances primitive endoderm-specific gene expression (43). YAP1 and TEADS reportedly increase the expression of pluripotency genes such as Oct3/4 and Sox2 $(43,44)$, as well as the trophoblast gene $C d x 2(45,46)$. However, levels of Oct $3 / 4$, SOX2, and CDX2 proteins were normal in our Mob1-deficient embryos. Thus, although we found YAP1 to be activated in the 
A
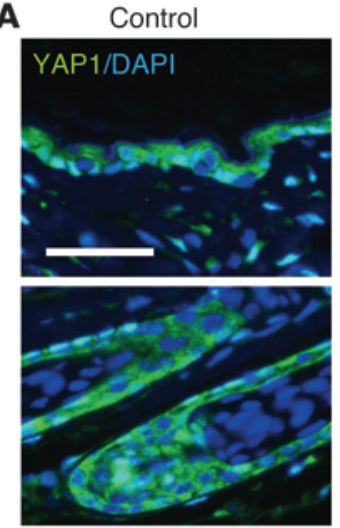

D
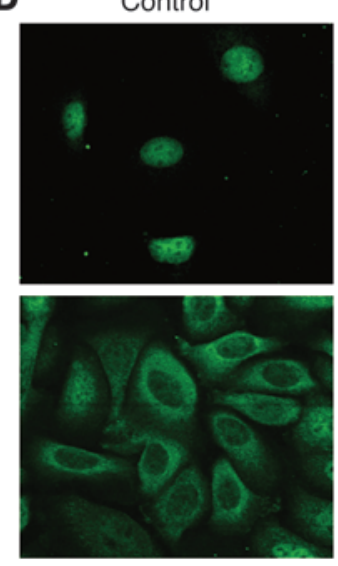

$k \mathrm{DKO}(\mathrm{P} 1)$

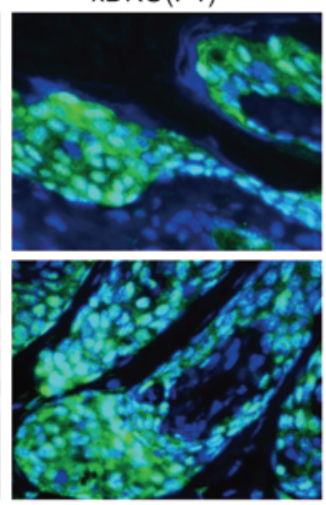

$k \mathrm{DKO}(\mathrm{P} 1)$

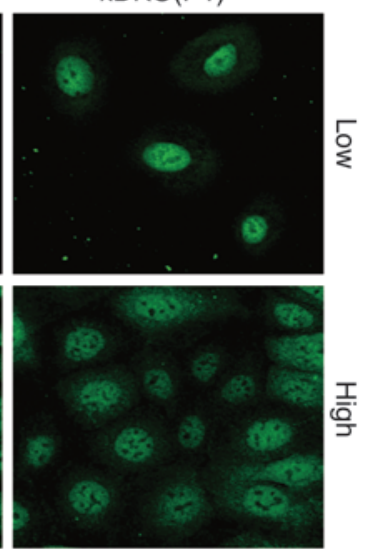

B

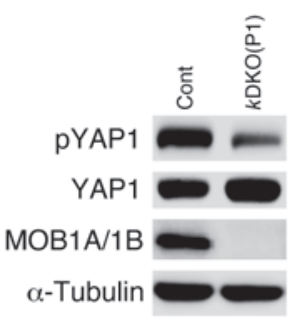

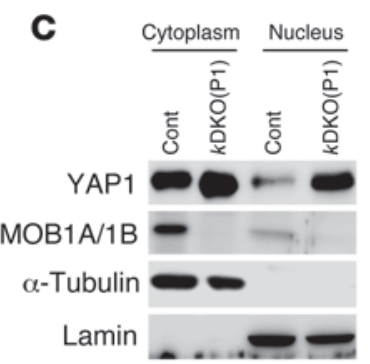

$\mathbf{E}$

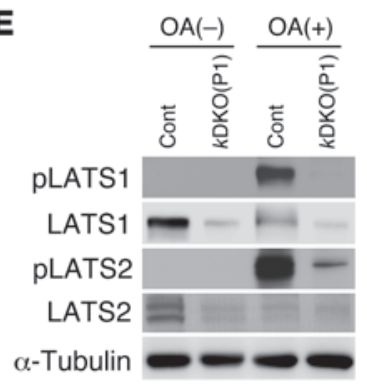

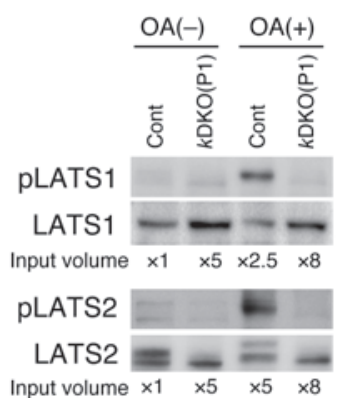

Figure 5

MOB1-mediated regulation of the LATS1/2-YAP1 pathway controls skin homeostasis. (A) Immunostaining of keratinocytes from control and $k \mathrm{DKO}(\mathrm{P} 1)$ mice at P19 to detect YAP1 in the IFE (top) and HF (bottom). Scale bar: $50 \mu \mathrm{m}$. (B) Immunoblot of total extracts of control and $k D K O(P 1)$ keratinocytes to detect the indicated proteins. $\alpha$-Tubulin, loading control. (C) Immunoblot of cytoplasmic and nuclear fractions of control and $k \mathrm{DKO}(\mathrm{P} 1)$ keratinocytes to detect the indicated proteins. $\alpha$-Tubulin and Lamin, cytoplasmic and nuclear loading controls, respectively. (D) Immunostaining to detect YAP1 in keratinocytes from control and kDKO(P1) mice plated at low or high cell density. YAP1 is localized in the nucleus in mutant keratinocytes even at high cell density. (E) Immunoblot to detect the indicated proteins in total extracts of control (Cont) and $k D K O(P 1)$ keratinocytes that were left untreated (OA-) or treated with OA (OA+). Left: unadjusted lysates. Right: levels of LATS1 and LATS2 proteins in each sample were adjusted to equality before electrophoresis. Results shown are representative of at least 3 independent trials and at least 3 mice/group.

mutant ICM, a much stronger alteration of YAP1 activity may be necessary to induce a detectable effect on these genes.

We have shown that, in contrast to MST1/2 and LATS1/2, MOB1 is a potent tumor suppressor in a range of tissue types that includes the skin. All cancers examined in Mob1 $a^{\Delta /+} 1 b^{t r / t r}$ and Mob1 $a^{\Delta / \Delta} 1 b^{t r /+}$ mice had lost the WT Mob1 allele, suggesting increased genetic instability. Mob1a/1b mRNAs and proteins are frequently cosuppressed in tumor cell lines (data not shown), and the MOB1A and MOB1B amino acid sequences are 95\% identical. We are currently clarifying whether a common mechanism regulates these 2 genes.

In our Mob1 $a^{\Delta /+} 1 b^{t r / t r}$ and Mob1 $a^{\Delta / \Delta} 1 b^{t r /+}$ mice, the most frequent tumors were malignant outer root sheath tumors resembling trichilemmal carcinomas (Figure 6A). Histologically, these malignancies were not BCCs because they lacked the cellular palisading typical of BCCs (4). Moreover, cultured Mob1a/1b double-mutant keratinocytes did not show the SHH pathway activation important for BCC onset (Figure 6C). Benign trichilemmomas are frequently observed in Cowden disease patients with hereditary PTEN muta- tions (47), but these tumors seldom become malignant. The trichilemmal growths in our Mob1-deficient mutants were clearly cancerous but showed no activation of the PTEN effector AKT (Figure 6C). Importantly, like our mutant mouse tissues, our human trichilemmal carcinoma samples exhibited frequent MOB1A/1B inactivation and YAP1 activation (Figure 6G and Table 3 ). These findings suggest that impaired Hippo signaling may drive trichilemmal carcinoma onset in humans.

Mob1-deficient keratinocytes exhibit enhanced proliferation, apoptotic resistance, impaired contact inhibition, increased centrosomes, accelerated mitotic exit, and enhanced progenitor selfrenewal. In addition, polarity must be defective without MOB1 because (a) KRT15 ${ }^{+}$cells were scattered inside the IFE and not localized to the basal layer; (b) CD34+ bulge stem cells were not localized in the bulge; and (c) hair bundles in the organ of Corti were disorganized (Supplemental Figure 2B). To date, 2 transgenic mouse strains overexpressing Yap1 in the skin have been described $(23,48)$. Like our Mob1 mutants, Yap1 transgenic mice show hyperplastic IFE. However, these latter animals also have a severe defect in HF 
A
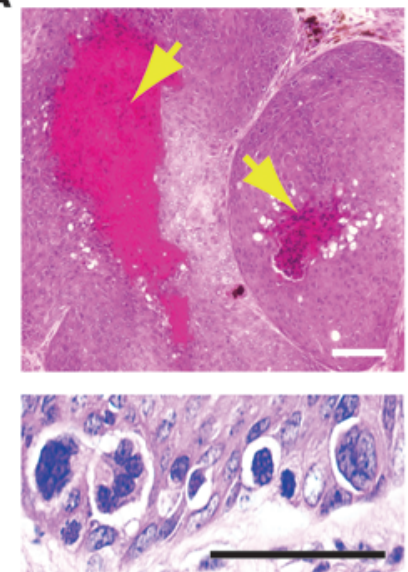

C
D Control

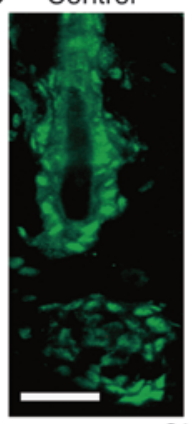

GLI2

E

GLI2(FL)

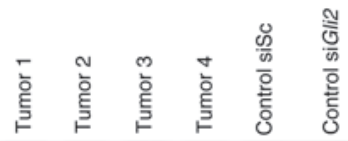

$\beta$-Actin

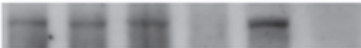

F

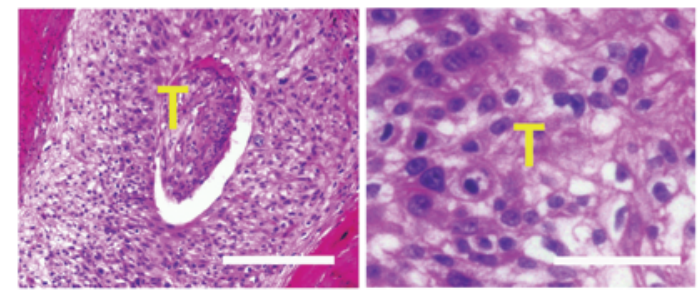

B
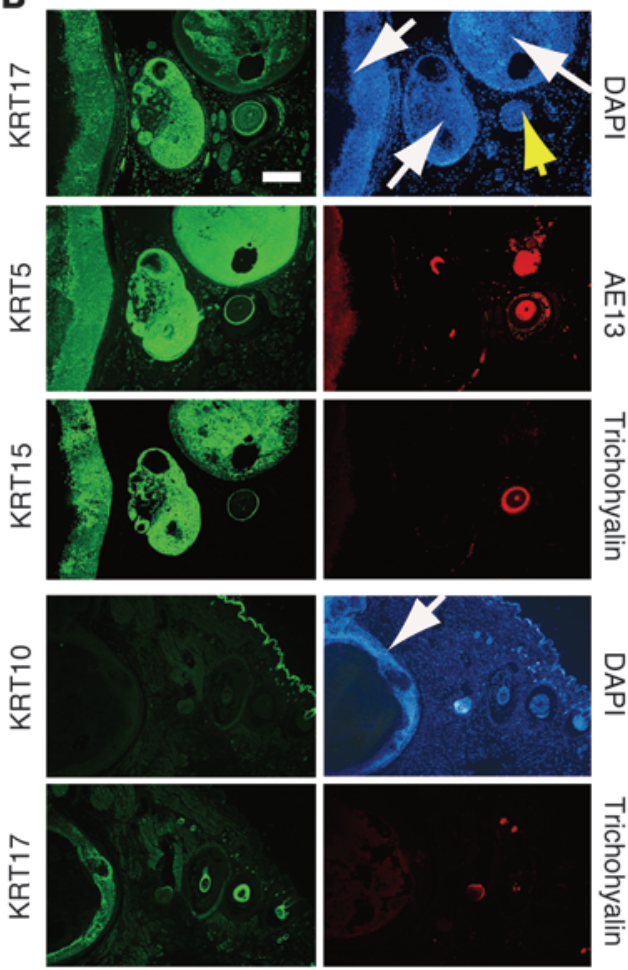

G
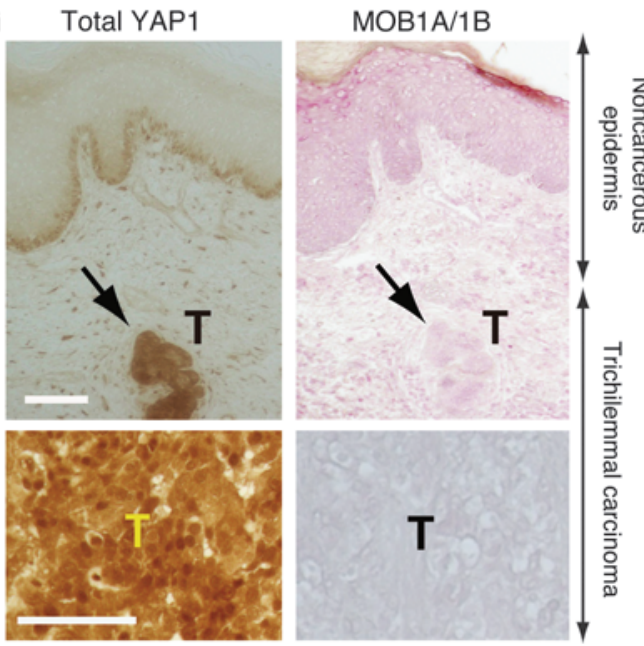

Figure 6

Characterization of skin cancers of Mob1-deficient mice and human trichilemmal carcinomas. (A) H\&E-stained sections of representative tumors from Mob1 $a^{\Delta / \Delta} 1 b^{t r /+}$ or Mob1 $a^{\Delta /+1} b^{t r / t r}$ mice showing: (top left) characteristic trichilemmal keratinization (yellow arrows; also in right); (bottom left) atypical and highly mitotic cells; and (right) continuity with the epidermis. Scale bars: $50 \mu \mathrm{m}$ (left); $500 \mu \mathrm{m}$ (right). (B) Immunostaining of tumors from Mob1 $a^{\mathrm{N} \Delta} \mathrm{b}^{\mathrm{tr} / \mathrm{t}}$ or Mob1 $\mathrm{a}^{\mathrm{N}+1} \mathrm{~b}^{\mathrm{tr} / \mathrm{tr}}$ mice to detect the indicated skin markers. White arrows, cancerous lesions; yellow arrow, nontumorous HF. Scale bar: $200 \mu \mathrm{m}$. (C) Immunoblot of total extracts of control and $k D K O(P 1)$ keratinocytes to detect ERK and AKT activation as well as the HF morphogenesis proteins GLI2 (FL; full length), LEF1 and HES1. $\beta$-Actin, loading control. (D) Immunostaining to detect GLI2 in HFs from control or $k D K O(P 1)$ mice. Scale bar: $25 \mu \mathrm{m}$. (E) Immunoblot to detect GLI2 in total extracts of control keratinocytes transfected with scramble siRNA (Control siSc) or Gli2 siRNA (Control siGli2), as well as in samples of 4 trichilemmal carcinomas (Tumor 1, 2, 3, 4) from kDKO(P1) mice. (F) H\&E staining of a human trichilemmal carcinoma viewed at low (scale bar: $200 \mu \mathrm{m}$ ) or high magnification (scale bar: $50 \mu \mathrm{m}$ ). (G) Immunostaining to detect YAP1 and MOB1A/1B in human trichilemmal carcinomas and nearby noncancerous tissues. Top: Both noncancerous and trichilemmal carcinoma $(T)$ tissues can be seen in low-magnification images. Bottom: High-magnification images of human trichilemmal carcinomas. Scale bars: $100 \mu \mathrm{m}$. Results shown are representative of at least 3 independent trials and at least 3 mice/group. 


\section{Table 3}

Quantitation of human trichilemmal carcinoma samples showing an increase in YAP1 + cells and/or elevation in nuclear YAP1, and a decrease in MOB1A/1B

\begin{tabular}{cccc} 
& $\begin{array}{c}\text { Increased } \\
\text { YAP1 }\end{array}$ & $\begin{array}{c}\text { Nuclear accumulation } \\
\text { of YAP1 }\end{array}$ & $\begin{array}{c}\text { Decreased } \\
\text { MOB1A/1B }\end{array}$ \\
Trichilemmal carcinoma & $11 / 14(79 \%)$ & $10 / 14(71 \%)$ & $5 / 10(50 \%)$ \\
\hline
\end{tabular}

\section{Methods}

Generation of Mob1 aflox and Mob1 $b^{\text {trap }}$ mice. A conditional targeting vector based on the Cre-loxP system was constructed to delete a genomic fragment containing exon 2 of the murine Mob1a gene (Supplemental Figure 1). This deletion generates a frame shift and early stop codon in exon 3. We introduced 1 loxP site into Mobla intron 1 and 2 loxP sites into intron 2 to flank Mob1a exon 2. The $P G K-H y g$ resistance cassette was inserted in antisense orientation between the 2 loxP sites in intron 2 . The resulting

formation. This difference may account for the development of IFE-derived squamous cell carcinomas in Yap1 transgenic mice, but HF-derived trichilemmal carcinomas in Mob1-deficient mutants.

At the biochemical level, Mob1-deficient keratinocytes exhibited reductions in not only phospho-LATS1/2, but also total LATS1/2 proteins. Lats $1 / 2$ mRNAs were comparable in control and mutant keratinocytes (data not shown), indicating that this surprising decrease in LATS $1 / 2$ proteins occurs posttranscriptionally. Like MOB1, SAV1 is a scaffolding protein in the Hippo pathway, but unlike Mob1 deficiency, Sav1 deficiency decreases phospho-MST, but does not affect total MST or LATS1/2 proteins (20). Another interesting biochemical observation was the activation of ERK in our Mob1-deficient keratinocytes: no connection between MOB1 and ERK has been reported to date. With respect to Hippo signaling, ERK can be activated by YAP1 (49) or suppressed by NF2 (50) or MST2 (51). Since NF2 and phospho-MST1/2 were not decreased in our Mob1-deficient keratinocytes (data not shown), we speculate that the increased YAP1 in these cells may have triggered their abnormal ERK activation.

Because all of our Mob1a/1b-deficient mice spontaneously developed tumors (and especially trichilemmal carcinomas), we believe that the loss of MOB1A/1B helps to both initiate and promote carcinoma onset. With respect to tumor initiation, the observed increase in the number of centrosomes and/or micronuclei in our mutant cells may have introduced detrimental alterations into their DNA. With respect to cancer promotion, the enhanced proliferation, apoptotic resistance, impaired contact inhibition, and increased progenitor self renewal associated with loss of MOB1A/1Bmay support the growth and progression of cells that have undergone tumor initiation events.

The very rare occurrence of trichilemmal carcinomas in humans has slowed the identification of genes involved in their development. Expression levels of MOB1A, LATS1/2, and SAV1 are more frequently reduced in the advanced stages of colon (33), breast (52), and renal cancers (53) than in the early stages of these malignancies. In lung cancers, the reverse is true, since MOB1A levels are frequently lower in the early pT1 stage of nonsmall-cell lung cancer as compared with later stages (34). These observations suggest that the loss of Hippo signaling molecules can be an important driver of cancer progression in humans. Further study of alterations to gene or protein expression or functions of Hippo signaling components in a broad range of human malignancies may increase our understanding of their involvement in tumorigenesis.

In conclusion, our results demonstrate that (a) MOB1 is a broadly-acting tumor suppressor in mice and (b) Hippo signaling drives trichilemmal carcinoma onset in the skin. Therapeutic strategies to control Hippo signaling or MOB1 expression might therefore benefit many cancer patients, particularly those with HF-derived cancers. targeting vector was electroporated into E14K ES cells, and homologous recombinants were confirmed by Southern blotting using 5 ' flanking probe A and a Hyg-specific probe. Correctly targeted Mob1a mutant clones were transiently transfected with pMC1-Cre (54) to delete the loxP-flanked $H y g^{r}$ gene. Progeny clones sensitive to hygromycin were subjected to Southern blotting to identify those retaining exon 2 flanked by 2 loxP sites (the Mob1 $a^{\text {flox }}$ allele) and those lacking exon $2\left(M o b 1 a^{\Delta}\right.$ allele; equivalent to a knockout mutation). For Mob1b-deficient mice, an ES cell line bearing a gene trap mutation of intron 2 of the Mob1b gene was obtained from the Sanger Institute (Hinxton, United Kingdom). The single integration of the trapped site was confirmed by PCR and genomic Southern blotting using $5^{\prime}$ flanking probe $\mathrm{C}$ and a neospecific probe. Mob1 $1 a^{f l o x}, M o b 1 a^{\Delta}$, and $M o b 1 b^{\text {trap }}$ mice were generated from ES cell clones using standard procedures and backcrossed to C57BL/ 6 mice 3 times before intercrossing to generate Mob1 $a^{f / f l}, M o b 1 a^{\Delta / \Delta}$, and Mob1 $b^{t r / t r}$ progeny. Primers used for genotyping are listed in Supplemental Table 2.

In vitro culture of preimplantation embryos. E3.5 embryos derived from the intercrossing of $M o b 1 a^{\Delta /+} 1 b^{t r / t r}$ males and females were analyzed as described (55). Briefly, blastocysts were individually cultured in 24-well plates in ES cell medium without LIF and photographed every 24 hours. On day 8 , the morphology of each embryo was recorded and its genotype determined by PCR.

Embryo immunostaining. Immunofluorescent staining of embryos was performed as described (45). Briefly, embryos (E3.5) were cultured overnight prior to fixation in $4 \%$ paraformaldehyde. Fixed tissues were permeabilized with $0.2 \%$ Triton $\mathrm{X}-100$, blocked with $2 \%$ goat serum, and incubated overnight at $4{ }^{\circ} \mathrm{C}$ with Abs recognizing CDX2 (Biocare Medical), Oct $3 / 4$ (gift of H. Niwa, Riken), PDGFR $\alpha$ (eBiosciences), GATA4/6 (Santa Cruz Biotechnology Inc.), NANOG (ReproCELL), Troma-1 (DSHB), SOX2 (Abcam), or YAP1 (Sigma-Aldrich). Secondary Abs were Alexa Fluor 488 goat anti-rabbit or Alexa Fluor 568 goat anti-mouse (Molecular Probes). Nuclei were visualized using DAPI (Dojindo). Stained embryos were examined by confocal microscopy.

Generation and differentiation of Mob1a/1b double-mutant ES cells. ES cells established from embryos derived from Mob1 $1 a^{f / f l} 1 b^{t r / t r}$ intercrosses were transfected with linearized CMV-Mer-Cre-Mer plasmid DNA (56) plus pApuro2 (57). To generate ERCreMob1 $a^{f l / f l} 1 b^{\text {tr/tr }}$ ES clones, ES cells were selected by culture for 12 days in complete ES cell medium containing $1 \mu \mathrm{g} / \mathrm{ml}$ puromycin (Invivogen) on an irradiation-inactivated Puro MEF feeder layer. Tamoxifen ( $3.3 \mu \mathrm{M}$; Toronto Research Chemicals) was added (or not) for 2 days to completely delete the Mob1a gene (data not shown). Control and mutant EBs were generated from hanging drops of approximately 1,000 ES cells $/ 20 \mu 1$ medium as described (58).

Generation of keratinocyte-specific Mob1a/Mob1b double-mutant mice. Mob1 al/fll $1 b^{\text {tr/tr }}$ mice were mated to Krt14CreER transgenic mice (35) in which Cre can be activated by tamoxifen under the control of the Krt14 promoter. Offspring carrying Krt14CreER plus 2 copies of the floxed Mob1a allele (Mob1 $\left.a^{l / f l}\right)$ and 2 copies of the trapped Mob1b allele (Mob1 $\left.b^{t r / t r}\right)$ were administered tamoxifen and used in the analysis as homozygous double mutant (Krt14CreERMob1afl/fl $1 b^{t r / t r}+$ tamoxifen; $k \mathrm{DKO}$ ) mice. 
Krt14CreERMob1 $a^{f / f l} 1 b^{t r / t r}$ mice without tamoxifen and Mob1 $a^{+/+} 1 b^{t r / t r}$ or $M o b 1 a^{+/+}$Mob1 $1 b^{t r / t r}$ mice with tamoxifen but without Krt14CreER were indistinguishable in pilot experiments examining histology and MOB1 protein levels. Krt14CreERMob1 $a^{f l / f l} 1 b^{\text {tr/tr }}$ mice without tamoxifen were arbitrarily chosen to serve as controls. To induce Cre in keratinocyte stem/progenitor cells, P1 mice were intraperitoneally administered 0.2 $\mathrm{mg}$ tamoxifen on that one day, or P28 mice received $0.5 \mathrm{mg}$ tamoxifen each day for 7 days starting on P28.

Characterization of primary keratinocytes. Primary keratinocytes isolated from control and $k \mathrm{DKO}$ mice were cultured as described (59). For cell saturation density assays, keratinocytes at passage 2 were seeded in type I collagen-coated 24 -well plates $\left(0.3 \times 10^{5} /\right.$ well $)$ and cultured for up to 12 days before counting cell numbers. For colony-forming assays, keratinocytes at passage 1 were seeded in 6 -well plates $\left(0.4 \times 10^{4} /\right.$ well). After 2 weeks, half the wells were fixed and stained with Giemsa (Muto Pure Chemicals), and the number of primary colonies was counted. Keratinocytes of an unfixed well were then trypsinized and reseeded so that each well of a 6-well plate received cells from 1 primary colony. Reseeded cells were cultured for 2 weeks to generate secondary colonies.

Clinical samples. Surgically obtained trichilemmal carcinoma samples containing noncancerous tissues were acquired from the Department of Regenerative Dermatology (Osaka University). Resected cancer tissues were fixed in formalin and stained with anti-YAP1 $\mathrm{Ab}$ (Cell Signaling) and antiMOB1A/1B Ab (AP7031b; Abgent) using a standard protocol.

Statistics. KaleidaGraph software was used for statistical analyses. Data are shown as the mean $\pm \mathrm{SEM}$, and $P$ values were determined using the 2 -tailed Student's $t$ test unless otherwise stated. $P<0.05$ was considered statistically significant.

Study approval. The clinical sample study design was approved by the Institutional Review Board of Osaka University, and written informed consent was obtained from all patients. All animal experiments were approved by the Animal Experiment Review Boards of Kyushu University and Akita University.

See Supplemental Methods for additional details.

\section{Acknowledgments}

We are grateful to S. Kuroda, Y. Horie, M. Natsui, H. Takahashi, and K. Enomoto (all of Akita University); to M. Fukumoto (of Tohoku University); to H. Konishi, A. Fujimoto, M. Suzuki, C. Meno, and Y. Oda (all of Kyushu University); and to K. Katsuoka (of Kitasato University) for expert technical support and helpful discussions. In addition, we thank B. Vanhaesebroeck (Barts Cancer Institute, London, United Kingdom) for critical reading of the manuscript. This work was supported by grants from the Ministry of Education, Culture, Sports and Technology of Japan (MEXT); the Cooperative Research Project Program of the Medical Institute of Bioregulation, Kyushu University; the Uehara Memorial Foundation; the Takeda Medical Foundation; the Naito Foundation; the Sumitomo Foundation; the Ono Medical Research Foundation; the Yasuda Medical Foundation; the Cosmetology Research Foundation; the Osaka Cancer Research Foundation; the Fukuoka Foundation for Sound Health; and the Astellas Foundation for Research on Metabolic Disorders.

Received for publication March 8, 2012, and accepted in revised form September 13, 2012.

Address correspondence to: Akira Suzuki, Division of Cancer Genetics, Medical Institute of Bioregulation, Kyushu University, 3-1-1 Higashiku, Higashiku, Fukuoka, Fukuoka 812-8582, Japan. Phone: 81.92.642.6838; Fax: 81.92.632.1499; E-mail: suzuki@ bioreg.kyushu-u.ac.jp.
1. Blanpain C, Horsley V, Fuchs E. Epithelial stem cells: turning over new leaves. Cell. 2007;128(3):445-458.

2. Reya T, Morrison SJ, Clarke MF, Weissman IL. Stem cells, cancer, and cancer stem cells. Nature. 2001;414(6859):105-111.

3. Headington JT. Tumors of the hair follicle. A review. Am J Pathol. 1976;85(2):479-514.

4. Fan H, Oro AE, Scott MP, Khavari PA. Induction of basal cell carcinoma features in transgenic human skin expressing Sonic Hedgehog. Nat Med. 1997; 3(7):788-792.

5. Oro AE, Higgins KM, Hu Z, Bonifas JM, Epstein EH Jr, Scott MP. Basal cell carcinomas in mice overexpressing sonic hedgehog. Science. 1997;276(5313):817-821.

6. Edgar BA. From cell structure to transcription: Hippo forges a new path. Cell. 2006;124(2):267-273.

7. Harvey K, Tapon N. The Salvador-Warts-Hippo pathway - an emerging tumour - suppressor network. Nat Rev Cancer. 2007;7(3):182-191.

8. Hamaratoglu F, et al. The tumour-suppressor genes NF2/Merlin and expanded act through Hippo signalling to regulate cell proliferation and apoptosis. Nat Cell Biol. 2006;8(1):27-36.

9. Creasy CL, Chernoff J. Cloning and characterization of a human protein kinase with homology to Ste20. J Biol Chem. 1995;270(37):21695-21700.

10. Tao W, et al. Human homologue of the Drosophila melanogaster lats tumour suppressor modulates CDC2 activity. Nat Genet. 1999;21(2):177-181.

11. Valverde P. Cloning, expression, and mapping of hWW45, a novel human WW domain-containing gene. Biochem Biophys Res Commun. 2000; 276(3):990-998.

12. Moreno CS, Lane WS, Pallas DC. A mammalian homolog of yeast MOB1 is both a member and a putative substrate of striatin family-protein phosphatase 2A complexes. J Biol Chem. 2001;276(26):24253-24260.

13. Sudol M, et al. Characterization of the mammalian
YAP (Yes-associated protein) gene and its role in defining a novel protein module, the WW domain. J Biol Chem. 1995;270(24):14733-14741.

14. St John MA, et al. Mice deficient of Lats1 develop soft-tissue sarcomas, ovarian tumours and pituitary dysfunction. Nat Genet. 1999;21(2):182-186.

15. Zhou D, et al. Mst 1 and Mst 2 maintain hepatocyte quiescence and suppress hepatocellular carcinoma development through inactivation of the Yap1 oncogene. Cancer Cell. 2009;16(5):425-438.

16. Tapon N, et al. Salvador promotes both cell cycle exit and apoptosis in Drosophila and is mutated in human cancer cell lines. Cell. 2002;110(4):467-478.

17. Lai ZC, et al. Control of cell proliferation and apoptosis by mob as tumor suppressor, mats. Cell. 2005; 120(5):675-685.

18. Pan D. The hippo signaling pathway in development and cancer. Dev Cell. 2010;19(4):491-505.

19. McClatchey AI, Saotome I, Ramesh V, Gusella JF, Jacks T. The Nf2 tumor suppressor gene product is essential for extraembryonic development immediately prior to gastrulation. Genes Dev. 1997; 11(10):1253-1265.

20. Lee JH, et al. A crucial role of WW45 in developing epithelial tissues in the mouse. EMBO J. 2008; 27(8):1231-1242.

21. McPherson JP, et al. Lats $2 / \mathrm{Kpm}$ is required for embryonic development, proliferation control and genomic integrity. EMBO J. 2004;23(18):3677-3688.

22. Morin-Kensicki EM, et al. Defects in yolk sac vasculogenesis, chorioallantoic fusion, and embryonic axis elongation in mice with targeted disruption of Yap65. Mol Cell Biol. 2006;26(1):77-87.

23. Schlegelmilch K, et al. Yap1 acts downstream of alpha-catenin to control epidermal proliferation. Cell. 2011;144(5):782-795.

24. Luca FC, Winey M. MOB1, an essential yeast gene required for completion of mitosis and mainte- nance of ploidy. Mol Biol Cell. 1998;9(1):29-46.

25. Luca FC, Mody M, Kurischko C, Roof DM, Giddings $\mathrm{TH}$, Winey $\mathrm{M}$. Saccharomyces cerevisiae Mob1p is required for cytokinesis and mitotic exit. Mol Cell Biol. 2001;21(20):6972-6983.

26. Bardin AJ, Amon A. Men and sin: what's the difference? Nat Rev Mol Cell Biol. 2001;2(11):815-826.

27. Yoshida S, Toh-e A. Regulation of the localization of Dbf2 and mob1 during cell division of saccharomyces cerevisiae. Genes Genet Syst. 2001;76(2):141-147.

28. Mah AS, Jang J, Deshaies RJ. Protein kinase Cdc15 activates the Dbf2-Mob1 kinase complex. Proc Natl Acad Sci U S A 2001;98(13):7325-7330.

29. Hergovich A, Hemmings BA. Hippo signalling in the G2/M cell cycle phase: Lessons learned from the yeast MEN and SIN pathways. Semin Cell Dev Biol. 2012;23(7):794-802.

30. Chow A, Hao Y, Yang X. Molecular characterization of human homologs of yeast MOB1. Int J Cancer. 2010;126(9):2079-2089.

31. Stavridi ES, et al. Crystal structure of a human Mob1 protein: toward understanding Mob-regulated cell cycle pathways. Structure. 2003;11(9):1163-1170.

32. Bothos J, Tuttle RL, Ottey M, Luca FC, Halazonetis TD. Human LATS1 is a mitotic exit network kinase. Cancer Res. 2005;65(15):6568-6575.

33. Kosaka Y, Mimori K, Tanaka F, Inoue H, Watanabe M, Mori M. Clinical significance of the loss of MATS1 mRNA expression in colorectal cancer. Int J Oncol. 2007;31(2):333-338.

34. Sasaki H, et al. Human MOB1 expression in nonsmall-cell lung cancer. Clin Lung Cancer. 2007; 8(4):273-276

35. Vasioukhin V, Degenstein L, Wise B, Fuchs E. The magical touch: genome targeting in epidermal stem cells induced by tamoxifen application to mouse skin. Proc Natl Acad Sci US A. 1999;96(15):8551-8556.

36. Hardy MH. The secret life of the hair follicle. Trends 
Genet. 1992;8(2):55-61.

37. Yabuta $\mathrm{N}$, et al. Lats 2 is an essential mitotic regulator required for the coordination of cell division. J Biol Chem. 2007;282(26):19259-19271.

38. Hao Y, Chun A, Cheung K, Rashidi B, Yang X. Tumor suppressor LATS1 is a negative regulator of oncogene YAP. J Biol Chem. 2008;283(9):5496-5509.

39. Hirabayashi S, et al. Threonine 74 of MOB1 is a putative key phosphorylation site by MST2 to form the scaffold to activate nuclear Dbf2-related kinase 1. Oncogene. 2008;27(31):4281-4292.

40. Mill P, et al. Sonic hedgehog-dependent activation of Gli2 is essential for embryonic hair follicle development. Genes Dev. 2003;17(2):282-294.

41. Ewing RM, et al. Large-scale mapping of human protein-protein interactions by mass spectrometry. Mol Syst Biol. 2007;3:89.

42. Rual JF, et al. Towards a proteome-scale map of the human protein-protein interaction network. Nature. 2005;437(7062):1173-1178

43. Tamm C, Bower N, Anneren C. Regulation of mouse embryonic stem cell self-renewal by a YesYAP-TEAD2 signaling pathway downstream of LIF. J Cell Sci. 2011;124(pt7):1136-1144.

44. Lian I, et al. The role of YAP transcription coactivator in regulating stem cell self-renewal and differentiation. Genes Dev. 2010;24(11):1106-1118.

45. Nishioka N, et al. The Hippo signaling pathway components Lats and Yap pattern Tead 4 activity to distinguish mouse trophectoderm from inner cell mass. Dev Cel. 2009;16(3):398-410.

46. Yagi R, et al. Transcription factor TEAD4 specifies the trophectoderm lineage at the beginning of mammalian development. Development. 2007; 134(21):3827-3836

47. Farooq A, Walker LJ, Bowling J, Audisio RA. Cowden syndrome. Cancer Treat Rev. 2010;36(8):577-583.

48. Zhang H, Pasolli HA, Fuchs E. Yes-associated protein (YAP) transcriptional coactivator functions in balancing growth and differentiation in skin. Proc Natl Acad Sci U S A. 2011;108(6):2270-2275.

49. Kang W, et al. Yes-associated protein 1 exhibits oncogenic property in gastric cancer and its nuclear accumulation associates with poor prognosis. Clin Cancer Res. 2011;17(8):2130-2139.

50. Lim JY, et al. Merlin suppresses the SRE-dependent transcription by inhibiting the activation of RasERK pathway. Biochem Biophys Res Commun. 2003; 302(2):238-245.

51. Kilili GK, Kyriakis JM. Mammalian Ste20-like kinase (Mst2) indirectly supports Raf-1/ERK pathway activity via maintenance of protein phosphatase-2A catalytic subunit levels and consequent suppression of inhibitory Raf-1 phosphorylation. J Biol Chem. 2010;285(20):15076-15087.

52. Takahashi Y, Miyoshi Y, Morimoto K, Taguchi T, Tamaki Y, Noguchi S. Low LATS2 mRNA level can predict favorable response to epirubicin plus cyclo- phosphamide, but not to docetaxel, in breast cancers. J Cancer Res Clin Oncol. 2007;133(8):501-509.

53. Matsuura K, et al. Downregulation of SAV1 plays a role in pathogenesis of high-grade clear cell renal cell carcinoma. BMC Cancer. 2011;11:523.

54. Gu H, Zou YR, Rajewsky K. Independent control of immunoglobulin switch recombination at individual switch regions evidenced through Cre-loxP-mediated gene targeting. Cell. 1993;73(6):1155-1164.

55. Suzuki A, et al. Brca2 is required for embryonic cellular proliferation in the mouse. Genes Dev. 1997; 11(10):1242-1252.

56. Verrou C, Zhang Y, Zurn C, Schamel WW, Reth M. Comparison of the tamoxifen regulated chimeric Cre recombinases MerCreMer and CreMer. Biol Chem. 1999;380(12):1435-1438.

57. Takata M, et al. Tyrosine kinases Lyn and Syk regulate B cell receptor-coupled Ca2+ mobilization through distinct pathways. EMBOJ. 1994;13(6):1341-1349.

58. Sakai Y, Yoshiura Y, Nakazawa K. Embryoid body culture of mouse embryonic stem cells using microwell and micropatterned chips. J Biosci Bioeng. 2011;111(1):85-91.

59. Lichti U, Anders J, Yuspa SH. Isolation and shortterm culture of primary keratinocytes, hair follicle populations and dermal cells from newborn mice and keratinocytes from adult mice for in vitro analysis and for grafting to immunodeficient mice. Nat Protoc. 2008;3(5):799-810. 